\title{
Nonlinear Dynamic Analysis of New Product Diffusion considering Consumer Heterogeneity
}

\author{
Zhongjun Tang and Huike Zhu \\ Research Base of Beijing Modern Manufacturing Development, College of Economics and Administration, \\ Beijing University of Technology, No. 100 Pingleyuan, Beijing, 100124, China
}

Correspondence should be addressed to Huike Zhu; huikezhu@emails.bjut.edu.cn

Received 22 June 2020; Revised 5 August 2020; Accepted 11 September 2020; Published 22 September 2020

Academic Editor: Wei Zhou

Copyright (C) 2020 Zhongjun Tang and Huike Zhu. This is an open access article distributed under the Creative Commons Attribution License, which permits unrestricted use, distribution, and reproduction in any medium, provided the original work is properly cited.

\begin{abstract}
When a new product enters the market, individual consumers' decision-making behavior and purchase time are uncertain. Based on the dynamics of epidemic transmission theory and agent modeling technology, this study proposes a new coupling model through the combination of the improved SEIR epidemic model and the heterogeneous agent model. This model considers consumer heterogeneity resulting from three aspects in consumers' sensitivity, network topology, and considerations of information flow received. It aims to analyze how consumer heterogeneity affects the scale and speed of new product diffusion. The proposed model showed that consumers' characteristics and behavior combination at the microlevel lead to the diversity of nonlinear diffusion curves at the macrolevel for new products. Moreover, a pilot study is conducted to simulate this model and examine how to estimate the model's parameters using aggregated data about film products. The pilot study results suggested that different consumer characteristics and behavior combinations affect the scale and speed of new product diffusion to varying degrees. In different scenarios, there were significant differences in the influence of the degree of consumer heterogeneity on diffusion, accompanied by the occurrence of threshold. The results of the empirical analysis in this study are in line with reality.
\end{abstract}

\section{Introduction}

Successful introduction of new products to the market has become particularly important. High-quality and nearly perfect new products will appear with the phenomenon of promotion failure without diffusion of products, threatening the stable and rapid development of enterprises. In the process of new product diffusion, there are strong differences in consumers' sensitivity to the price, quality, and advertisement of new products; the way of communication; and the initial perception and perceived reliability of new product information. These differences make the diffusion trajectory complex and changeable, which increases the difficulty of prediction. It is not conducive to the new product managers to develop effective marketing strategy combination, which will easily lead to the failure of new product promotion. Therefore, the impact of consumer heterogeneity on new product diffusion cannot be ignored. Most, but not all, previous studies considered narrow consumer heterogeneity, namely, the introduction of partial consumers' characteristics. Considering the availability of data as well as consumer characteristic integrity, this study makes a comprehensive reference to the previous classification of consumer heterogeneity. The narrow definition of consumer heterogeneity indicates that consumers have certain differences in three aspects: consumer sensitivity, consumers' network topology, and consumers' performance in terms of receiving the information flow.

At the macrolevel, decades of diffusion research have successfully described how new products spread in society [1]. The Susceptible-Exposed-Infectious-Recovered (SEIR) epidemic model with the incubation period is often used to describe the diffusion process of new product information [2]. In this paper, the epidemic transmission model with incubation period is improved in two aspects. On the one hand, compared with the carriers in the process of virus transmission, consumers have choice and judgment in the process of new product diffusion, so it is urgent to improve 
the process of new product diffusion. On the other hand, the fundamental disadvantage of SEIR epidemic model, a convergent-level model, is that it assumes a homogeneous population and ignores the study of individual heterogeneity. Individual heterogeneity has a significant impact on the scale and speed of new product diffusion. The main impact occurs in the following three aspects. First, consumer sensitivity to new products interacts with the surrounding environment, and the levels of learning and understanding information change dynamically over time. Thus, enterprises need to constantly update their market strategies. Second, due to individual differences, the purchasing process curve of new products will show a variety of possibilities with the change in time. Third, consumer heterogeneity indicates that there are systematic differences in the time when different consumers buy new products, which leads to uncertainty in the time when new products are ignited and spread. In conclusion, consumer heterogeneity should be introduced to improve the model.

However, although the improved SEIR model can describe the dynamic characteristics of new product diffusion at the macroscopic level, it lacks explanatory power for the microscopic mechanism. It is still controversial whether it can truly reflect the behavior of heterogeneous individuals at the microlevel $[1,3]$, and the mathematical verification of the model is very challenging after adding consumer heterogeneity.

To overcome these limitations, this study introduces the heterogeneous agent model (HAM), which is a microscopic computing model used to simulate the simultaneous actions and interactions of agents with autonomous consciousness and heterogeneity to reproduce and predict complex phenomena $[4,5]$. The agents in the model must have four types of abilities: autonomy, social ability, reactivity, and initiative. Although HAMs allow rich behaviors to be presented at the microlevel and can draw aggregate-level conclusions from these microlevel behaviors, they are often criticized as toy models that cannot fully capture actual behaviors in real market environments, mainly due to the lack of an empirical basis [6]. Moreover, to establish a relationship between the macro- and micro-levels, as well as to reveal how individual characteristics and behaviors at the microlevel affect the diffusion scale, speed, and volatility of the overall purchase behavior of new products at the macrolevel, it is urgent to propose an empirically based coupling model combining the macro- and micro-levels. That is, this involves the combination of an empirically grounded agent-based model and other different diffusion models. This coupling model is one of the valuable future research directions advocated by Fabian et al. [4]. In this case, a HAM based on the Bass model is proposed. However, in this type of model, the agents' properties and decision-making behavior are relatively single, and there is no stage classification for the category and behavior of agents. Therefore, this study proposes a coupling model based on an improved SEIR model and a HAM.

The contributions of this study are reflected in three aspects. First, considering the heterogeneity of consumers, this study is the first to solve the problem of transforming the transition probability from a constant parameter to a function proposed by Wu and Duan [7] as a future research direction. Individual characteristics and behavior combination attributes are added to the function to analyze the influence of consumer heterogeneity on the new product diffusion scale and speed. Second, a new coupling model based on the combination of the improved SEIR model and HAM is proposed. The traditional SEIR epidemic model is improved upon and combined with the HAM, and an empirical simulation experiment is conducted. This model overcomes the defect of the macrodynamic model, which describes only the surface phenomena. At the same time, the coupling model can explain the nonlinear dynamic mechanism of new product diffusion at the microlevel and build a bridge between the macro- and micro-levels. Third, this study extends the theory of new product diffusion. For different types of new products, the theoretical model constructed in this study can be used as a basic model to discuss the results of individual consumption behavior as well as macrodiffusion.

The remainder of this paper is organized as follows. Section 2 provides a literature review of the definition and classification of consumer heterogeneity as well as new product diffusion-related models. Section 3 presents the detailed model-building process. In Section 4, the simulation process for the pilot study is presented. Section 5 describes the series of simulation results. Section 6 tests the sensitivity and validity of the model. Section 7 draws conclusions and points out this study's shortcomings and future research directions.

\section{Literature Review}

2.1. Perspective of Consumer Heterogeneity. Previous studies defined consumer heterogeneity in two ways. The first type is generalized consumer heterogeneity, wherein individual consumers have all relevant individual characteristics that can affect the adoption of new products [8]. Fay and Xie proposed that consumer heterogeneity means that consumers have some differences in their evaluation of perfectly symmetrical products [9]. Chen et al. [10] used the definition of Fay and Xie [9] for reference and applied it to nonperfectly symmetric products. The second type is consumer heterogeneity in a narrow sense, which includes some features of a consumer. This definition is based on the study of new product diffusion, which involves and refines a variety of consumer heterogeneity characteristics. These characteristics can be summarized into the following four types.

The first is a study on the heterogeneity of consumer sensitivity. Shim and Bliemel introduced consumers' advertising impression threshold and word-of-mouth impression threshold to analyze how individual consumers' characteristics and behaviors affect the speed and range of diffusion at the macrolevel [1]. Rand and Rust introduced consumers' sensitivity when examining wine price, quality, and advertising to explore how consumer heterogeneity affects buying behavior [11]. Yi and Ahn added the value and initial expectation of consumers about new products into the model to explore how they impact the effectiveness of expectation management during the new product diffusion process [12]. Song et al. concluded that consumers' sensitivity to the price, time, and preference of new products 
affected the time of new product adoption and consumers' choice of brand [13]. The second type is consumer heterogeneity caused by network topology. Lin and Chintagunta proposed a heterogeneous network model with a consciousness stage and a decision stage to explain the process of new product diffusion [2]. Jun et al. analyzed the influence of a healthy food consumer network structure on the spread of panic behavior related to food safety using the theory of network stochastic advantage [14]. Third, consumer heterogeneity is caused by information flow. Khashanah and Alsulaiman studied the impact of differences in consumer information perception on the simulated market volatility index [15].

The fourth is a pairwise combination of the above three types. It has been shown that heterogeneity in consumer sensitivity and consumer heterogeneity caused by the network topology structure increase the complexity of financial market behavior $[16,17]$. Meng et al. discussed the diffusion performance of green products in competitive markets under consumer heterogeneity caused by network topology and consumer sensitivity to price and quality [18]. Lin et al. found that heterogeneity in consumer preference and consumer heterogeneity caused by the network topology structure affect the speed of new product diffusion and the fluctuation of group decisions [19]. Stummer et al. considered consumer price, quality, advertising sensitivity, and consumer heterogeneity caused by network topology, and they analyzed the influence of the above heterogeneity on the robustness of the range and speed of new product diffusion [6]. Chatterjee and Eliashberg added consumer sensitivity to price, quality, and risk and considered consumer heterogeneity caused by information flow, such as consumers' differences in initial perception and perceived reliability of information, to explain the aggregation-level behavior of new product adoption at the individual level [20].

To sum up, previous studies only involved consumer heterogeneity under one category or two combinations. In this study, the combination of the above three categories is considered comprehensively according to the characteristics of new products.

2.2. Dynamic Model of New Product Diffusion. Many diffusion phenomena in human systems are analogous to the social contagion processes of infectious diseases, including word of mouth, imitation, and network externalities. From the diffusion of new products to the spread of rumors and financial panics, to the dynamics of infectious diseases and their formal models, there is a rich history in the social sciences [21-24]. In recent years, researchers have used dynamic models to study the diffusion of new products.

The first is a separate epidemic model. The research ideas and analytical methods of infectious disease transmission dynamics can be used for studying new product diffusion [25], and the classic new product diffusion model is similar to the epidemiological model. Lin et al. proposed a heterogeneous network epidemic model with a consciousness stage and a decision stage to explain the process of new product diffusion [2]. To study the diffusion of new products in the market, Jiang et al. proposed a new product diffusion model using epidemiological methods [26]. Shao and Hu extended the SIR epidemic model to the SIRB model by introducing a new class of consumer types, which provided an environment to investigate the diffusion of different new product types in social networks [27]. The second category is a combination of epidemiological models with other dynamic models. Fibich proved that diffusion is not described by the SIR model but a new model-the Bass-SIR model-which combines the Bass model of new product diffusion with the SIR epidemic model [28].

However, the above models are all convergent models, and their fundamental disadvantage is that they assume a homogeneous population. In addition, the aggregation model cannot distinguish the social network of one potential consumer from that of another, and so they must assume a fully connected social network [4]. Moreover, it is very challenging for the above model to analyze the dynamic mechanism of consumer heterogeneity on new product diffusion at the individual level. This study establishes an improved epidemiological model that considers consumer heterogeneity to solve the above defects.

2.3. A HAM for New Product Diffusion. In recent years, although research on macroaggregation models has become very popular, this type of model cannot accurately consider the characteristics and behavior of consumers, and the HAM can overcome this limitation through microlevel modeling. Rand and Rust proposed criteria based on agent modeling and provided the application and conditions of the model [11]. For a comprehensive overview of the diffusion model for new products based on heterogeneous agents and their advantages, see Kiesling et al. [3]. Although HAMs allow rich behaviors to be presented at the microlevel and aggregate-level conclusions can be obtained from these behaviors, they are often criticized as toy models that cannot fully capture actual behaviors in real market environments, mainly due to the lack of an empirical basis [6].

In this case, a HAM based on the Bass model is proposed to study the new product diffusion. Rand and Rust established the HAM first, in which agent characteristics are based on the Bass model and proposed strict guidance standards for such a coupling model [11]. Yi and Ahn established a HAM based on the Bass model to formalize and analyze how consumers' initial expectations of new products affect the interdependent process of product sales, consumer satisfaction, and word of mouth [12].

However, in the HAM based on the Bass model, the agent's attribute and behavior are relatively single, and the agent's classification and behavior are insufficient. Therefore, this study proposes a coupling model based on an improved SEIR model and a HAM. Rahmandad and Sterman developed a model combining the classical SEIR model with the HAM, which is a nonlinear and deterministic differential equation model [29]. The purpose was to discuss the situation in which the two models are used separately, thus providing a theoretical basis and foundation for the model proposed in this study. 


\section{Model Formulation}

3.1. Framework. Research on the dynamics of consumers' purchasing behavior for new products has been the focus of academia at the frontier. The consumer purchase decision process as a convergent diffusion model, similar to the spread of disease or information that does not involve deliberate decisions, limits consumers' consideration of the purchase determinants at the individual level and lacks an overall behavioral explanation. If only the agent-based model is used to explain the consumers' purchase decision and behavior at the individual level, there is a lack of clear consumer state transition process, and this model lacks generality outside the case. Therefore, to solve limitations of the two models, a new model is proposed to analyze the nonlinear dynamics of new product consumer decision-making behavior.

First, an improved SEIR infectious disease model is constructed based on consumer heterogeneity, referred to as the SMBQ model and shown using the outer yellow line in Figure 1 and detailed in Section 3.2. Furthermore, the HAM is constructed to explain the probability of individual consumer state change in the SMBQ model. The HAM is shown in Figure 1 in the inner black frame and detailed in Section 3.3. Finally, a coupling model based on a combination of the SMBQ model and HAM is obtained. The model framework is shown in Figure 1.

3.2. SMBQ Model Based on Consumer Heterogeneity. Based on the traditional SEIR model and its improved SCIM risk contagion model [7], this section considers consumer heterogeneity to improve the basic SEIR epidemic model. The existence of consumer heterogeneity enables each consumer to have purchase probability, which means that the probability of an individual consumer state transition will no longer be a constant parameter but a function of dynamic changes over time. Assume that there are four types of consumers in the purchase process of a new product, which are defined as follows:

(1) Target consumer: when a new product enters the market, these consumers may receive some basic information such as price and product attributes, but they have not yet received basic information.

(2) Potential consumer: these consumers have received some basic information, but they cannot make a decision whether to buy or not based on this information. They need to collect more information through online and offline channels about the new product to become purchasing consumers at a certain time in the future.

(3) Purchasing consumer: when a new product enters the market, these consumers make purchasing decisions in the process of constantly gathering learning information.

(4) Abandonment consumer: when a new product enters the market, these consumers give up purchasing decision in the process of constantly gathering learning information.

These four types of agents are abbreviated as $S, M, B$, and $Q$, respectively. Based on the assumptions of previous research, the following assumptions are made about consumer behavior under heterogeneous conditions. (1) When a new product enters the market, each autonomous target consumer will make a decision based on the first stimulus of mass media or corporate promotion; this is considered as the passive learning decision stage. (2) When target consumers are exposed to new product news, some consumers' purchase decisions are uncertain, and there is a lingering period. (3) Potential consumers exhibit autonomous behaviors and are susceptible to interaction and conformity effects. Agents actively learn to collect more information about the new product online and offline, which is conducive to making decisions on whether to buy or not. (4) Consumers are heterogeneous, and the probability of each consumer's state transition changes dynamically over time. (5) Consumers have only one chance to buy new products and do not consider repeated purchase behavior. (6) The sum of the consumer market at any time is constant, denoted by $N$.

Based on the above definitions and assumptions, the SMBQ model describes the dynamic process of new product consumer buying behavior, as shown in Figure 2 and formula (3):

$$
\begin{aligned}
& \left\{\begin{array}{l}
\frac{\mathrm{d} S(t)}{\mathrm{d} t}=-\sum_{i=1}^{S(t)}\left[X_{1}(i, t)+X_{0}(i, t)+X_{2}(i, t)\right], \\
\frac{\mathrm{d} M(t)}{\mathrm{d} t}=\sum_{i=1}^{S(t)} X_{0}(i, t)-\sum_{j=1}^{M(t)} P\left(t \mid \Gamma_{j}, \Psi_{j}\right)-\sum_{j=1}^{M(t)} \frac{1}{N} B(t), \Theta(t) \xi_{j} \delta_{j}-\sum_{j=1}^{M(t)} X_{3}(j, t), \\
\frac{\mathrm{d} B(t)}{\mathrm{d} t}=\sum_{i=1}^{S(t)} X_{1}(i, t)+\sum_{j=1}^{M(t)} P\left(t \mid \Gamma_{j}, \Psi_{j}\right)+\sum_{j=1}^{M(t)} \frac{1}{N} \Theta(t) \xi_{j} \delta_{j} B(t), \\
\frac{\mathrm{d} Q(t)}{\mathrm{d} t}=\sum_{i=1}^{S(t)} X_{2}(i, t)+\sum_{j=1}^{M(t)} X_{3}(j, t),
\end{array}\right. \\
& S(t)+M(t)+B(t)+Q(t)=N, \\
& s(t)+m(t)+b(t)+q(t)=1 .
\end{aligned}
$$




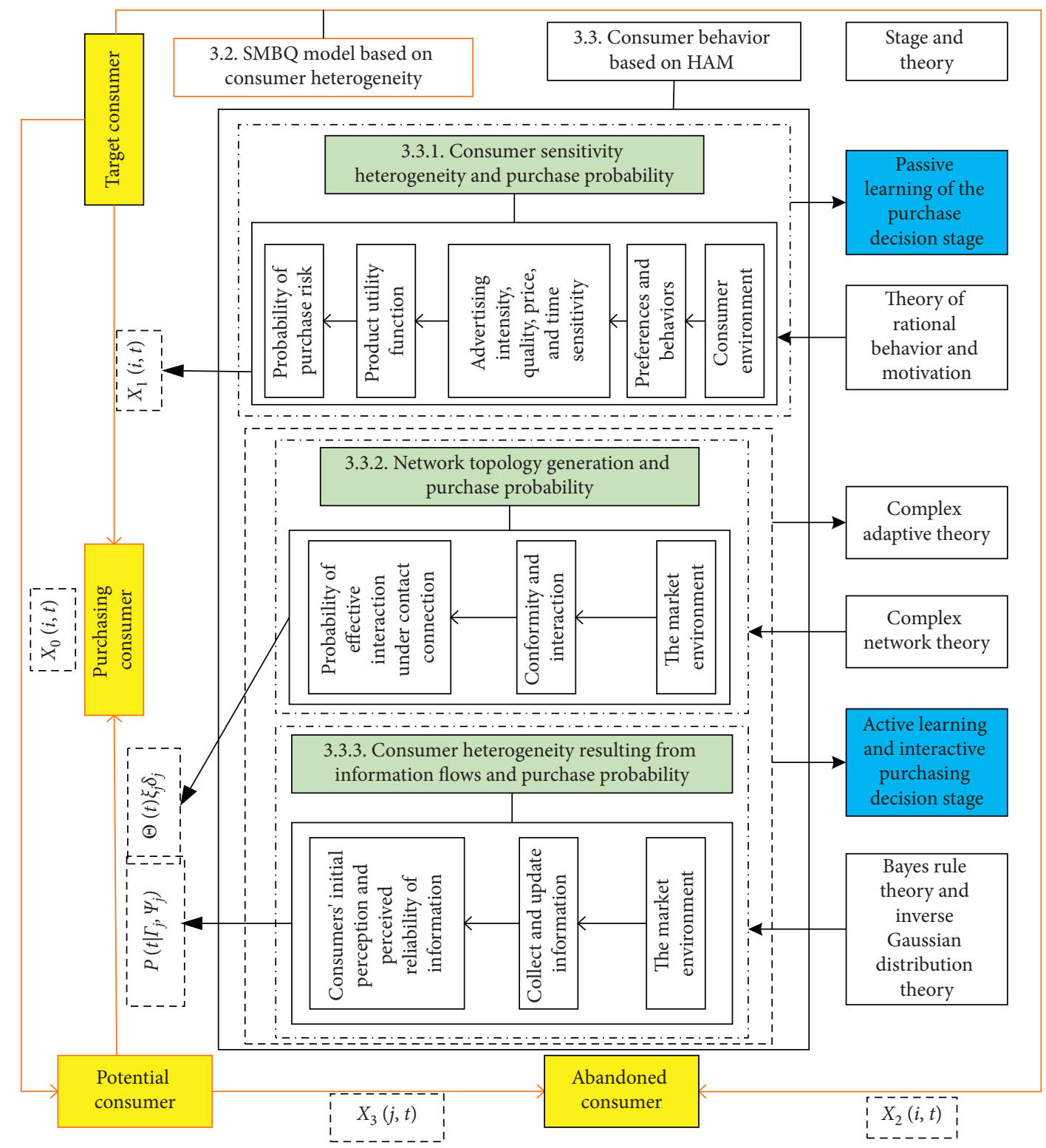

FIGURE 1: Coupling model framework based on a combination of the SMBQ model and the heterogeneous agent model (HAM).

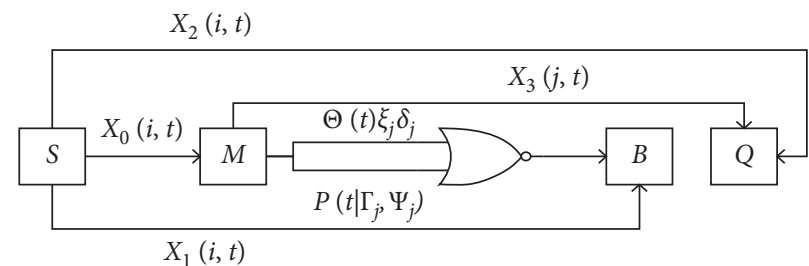

FIGURE 2: The dynamic process of consumer emergence behavior based on the SMBQ model. 
By dividing both sides of formula (1) by $N$ and substituting formula (2) into formula (1), we can conclude the following:

$$
\left\{\begin{array}{l}
\frac{\mathrm{d} s(t)}{\mathrm{d} t}=-\frac{1}{N} \sum_{i=1}^{\mathrm{Ns}(t)}\left[X_{1}(i, t)+X_{0}(i, t)+X_{2}(i, t)\right], \\
\frac{\mathrm{d} m(t)}{\mathrm{d} t}=\frac{1}{N}\left[\sum_{i=1}^{\mathrm{Ns}(t)} X_{0}(i, t)-\sum_{j=1}^{\mathrm{Nm}(t)} P\left(t \mid \Gamma_{j}, \Psi_{j}\right)-\sum_{j=1}^{\mathrm{Nm}(t)} \Theta(t) \xi_{j} \delta_{j} b(t)-\sum_{j=1}^{\mathrm{Nm}(t)} X_{3}(j, t)\right], \\
\frac{\mathrm{d} b(t)}{\mathrm{d} t}=\frac{1}{N}\left[\sum_{i=1}^{\mathrm{Ns}(t)} X_{1}(i, t)+\sum_{j=1}^{\mathrm{Nm}(t)} P\left(t \mid \Gamma_{j}, \Psi_{j}\right)+\sum_{j=1}^{\mathrm{Nm}(t)} \Theta(t) \xi_{j} \delta_{j} b(t)\right], \\
\frac{\mathrm{d} q(t)}{\mathrm{d} t}=\frac{1}{N}\left[\sum_{i=1}^{\mathrm{Ns}(t)} X_{2}(i, t)+\sum_{j=1}^{\mathrm{Nm}(t)} X_{3}(j, t)\right],
\end{array}\right.
$$

where $S(t), M(t), B(t)$, and $Q(t)$ represent the number of target consumers, potential consumers, purchasing consumers, and abandoning consumers, respectively, at time $t$. $s(t), m(t), b(t)$, and $q(t)$, respectively, represent the percentage of the above four categories of consumers among the total consumers. The meanings of the remaining parameters in the model are described in Table 1.

The left side of the first equation in system $(4) \mathrm{d} s(t) / \mathrm{d} t$ represents the population density of the target consumer at time $t$, and the negative sign on the right side of the first equation indicates that the target consumer is in the state of turning out. $(1 / N) \sum_{i=1}^{\mathrm{Ns}(t)}\left[X_{1}(i, t)+X_{0}(i, t)+X_{2}(i, t)\right]$ represents the population density of target consumers turning into potential consumers, purchasing consumers, and abandoning consumers at time $t$. That is to say, the first equation in system (4) says that the roll-out is equal to the roll-in. Similarly, the analysis of the remaining equations in system (4) will not be repeated here. At the same time, as $(\mathrm{d} s(t)) / \mathrm{d} t+(\mathrm{d} m(t)) / \mathrm{d} t+(\mathrm{d} b(t)) / \mathrm{d} t+(\mathrm{d} q(t)) / \mathrm{d} t=0$, the sum of $s(t)+m(t)+b(t)+q(t)=1$ is a constant. The above analyses indicate that system (4) is in equilibrium.

3.3. Consumer Behavior Based on the HAM. Based on the assumption of consumer heterogeneity, due to the consumers' adaptive ability, they constantly update their decisions with the change in the dynamic environment and interactions between consumers so that the transition probability between the states in the SMBQ model is no longer a constant parameter, but a function of a consumer and time $t$. Therefore, an individuallevel model is needed to explain the characteristics and behavior rules of consumers. The HAM provides a tool for understanding and analyzing this complex pattern. This section obtains the behavior rules and purchase decisions of individual consumers from three aspects: consumer sensitivity heterogeneity, consumer heterogeneity caused by network topology, and information flow.
3.3.1. Consumer Sensitivity Heterogeneity and Purchase Probability. According to rational behavior theory, a rational person supposes that most consumers act rationally [30]. Consumers make purchasing decisions based on the utility of the new product, which indicates the consumers' motivation and willingness to buy [18] and is the direct determinant of the consumers' purchase decision [31, 32]. Therefore, before constructing the model, it is assumed that the purchase process of new product consumers is based on the rational decision and motivation theory.

Assume that target consumer $i$ only considers one new product at time $t$, regardless of the impact of competing products. It is difficult to add all the attributes of new products and factors that can fully reflect the reality into the constructed model [31]. Therefore, it is assumed that only some key factors influencing consumers' purchase decisions are considered. The marketing theory states that the key incentives for consumers to make purchase decisions include price, quality, and advertising. Consumers' personality characteristics determine how these external stimuli simultaneously affect their decisions [31, 33]. At the same time, time pressure has an impact on consumers' decisionmaking behavior when purchasing new products [34, 35]. According to Zhang and Zhang [36], the improved utility function $U_{1 \text { it }}$ brought to target consumer $i$ by a single new product can be expressed by the following:

$$
U_{\text {lit }}=\chi_{\mathrm{it}} p_{\mathrm{it}}+\varepsilon_{\mathrm{it}} L_{t}+\phi_{\mathrm{it}} Q_{t}+\varphi_{\mathrm{it}} d_{t}
$$

where utility $U_{1 \text { it }}$ is affected by four factors. Subscript 1 represents the utility obtained by consumers when they buy new products, and $U_{0 \text { it }}$ represents the utility obtained by target consumer $i$ when the consumer does not buy new products at time $t$. $\chi_{\text {it }}$ represents the sensitivity parameter of target consumer $i$ to the price of new products at time $t . p_{\text {it }}$ is the price of the new product at time $t$. $\varepsilon_{\text {it }}$ represents the sensitivity parameter of target consumer $i$ to the advertising 
TABLE 1: Description of the parameters and functions used in the model.

\begin{tabular}{|c|c|}
\hline $\begin{array}{l}\text { Parameter/ } \\
\text { Function }\end{array}$ & Description \\
\hline$N$ & Total number of consumers in the new product market \\
\hline$\xi_{j}$ & Conformity degree of potential consumer $j$ \\
\hline & Degree of interaction between potential consumer $j$ and its neighbors \\
\hline$X_{0}(i, t)$ & Probability of target consumer $i$ changing into a potential consumer in the case of sensitivity heterogeneity at time $t$ \\
\hline$X_{1}(i, t)$ & Probability of target consumer $i$ changing into a purchasing consumer in the case of sensitivity heterogeneity at time $t$ \\
\hline$X_{2}(i, t)$ & $\begin{array}{c}\text { Probability of target consumer } i \text { changing into an abandoning consumer in the case of sensitivity heterogeneity at } \\
\text { time } t\end{array}$ \\
\hline$X_{3}(j, t)$ & $\begin{array}{c}\text { Probability that the potential consumer } j \text { will change into an abandoning consumer in the case of consumer } \\
\text { heterogeneity caused by network topology and information flow at time } t\end{array}$ \\
\hline$P\left(t \mid \Gamma_{j}, \Psi_{j}\right)$ & $\begin{array}{l}\text { Probability that the potential consumer } j \text { will change into a purchasing consumer in the case of consumer } \\
\text { heterogeneity caused by information flow at time } t\end{array}$ \\
\hline$\Theta(t)$ & Probability that potential consumers with the degree of $k$ at time $t$ have $n$ buying consumer neighbors \\
\hline
\end{tabular}

intensity of the new product at time $t$, which is subject to normal distribution. $L_{t}$ refers to the advertising intensity of the new product at time $t$. $\phi_{\text {it }}$ represents the sensitivity parameters of target consumer $i$ to the quality of new products. $Q_{t}$ refers to the quality of new products. $\varphi_{\text {it }}$ represents the sensitivity parameters of target consumer $i$ to the new product sales period at time $t$, which is subject to normal distribution. $d_{t}$ represents the dummy variable of the sales period of the new product. Finally, 1 represents the nonworking period, and 0 represents the working period.

Kim and Rossi first proposed that the price sensitivity of consumers is an exponential function of the difference between the actual price of a product and the expected price [37], which has been adopted by many scholars [18, 31, 36]. In this paper, by referring to this functional relation, the formula for the price sensitivity of consumers $\chi_{\mathrm{it}}$ is as follows:

$$
\chi_{\mathrm{it}}=-\hbar_{i}^{p_{\mathrm{it}}-\tilde{p}}+\mathfrak{\Im}
$$

where $\hbar_{i}$ is a parameter. The effect of price on consumers' utility is inverse. That is, as the price increases, consumers' utility decreases. If the above facts are met, equation (6) is a monotonically decreasing function. The negative sign in equation (6) changes the monotonicity of the function. According to the monotonicity of the exponential function, it can be known that $\hbar_{i}>1$, and $\mathfrak{I}$ is a constant (the value of $\mathfrak{I}$ is based on the socioeconomic attributes of the consumer). It is assumed that income represents all economic attributes of the consumer. $\widetilde{p}$ represents the expected price of consumers.

When consumers purchase new products, the closer the quality of the new product is to the expected quality of the products, the more sensitive the consumers are to the quality of the new products [31]. Therefore, expression $\phi_{i}$ is as follows:

$$
\phi_{\text {it }}=\lambda_{i}^{\left|Q_{t}-\widetilde{Q}\right|}+\Re
$$

where $\lambda_{i}$ is a parameter so that $0<\lambda_{i}<1, \mathfrak{R}$ is a constant (the value of $\mathfrak{R}$ is based on the socioeconomic attributes of the consumer), and it is still assumed that income represents all the economic attributes of the consumer. $\widetilde{Q}$ represents the expected quality of target consumer $i$.

According to Song and Chintagunta [13], the utility gained by target consumer $i$ without purchasing new products at time $t$ is

$$
U_{0 i t}=\log \left[\exp \left(U_{0 i, t-1}\right)+\exp \left(U_{1 i, t-1}\right)\right] .
$$

Furthermore, the risk rate of purchasing by target consumer $i$ at time $t$ is obtained as follows:

$$
h_{i, t}=\frac{1}{\left\{1+\exp \left(U_{0 \mathrm{it}}-U_{1 \mathrm{it}}\right)\right\}}
$$

For target consumer $i$ at time $t, h_{0 \text { it }}$ represents the risk rate of giving up the purchase, and the probability of target consumers buying new products is

$$
X_{g}(i, t)=\left\{\prod_{\tau=1}^{t-1} h_{0 \mathrm{it}}\right\} h_{\mathrm{it}}=\left\{\prod_{\tau=1}^{t-1}\left(1-h_{\mathrm{it}}\right)\right\} h_{\mathrm{it}} \text {, }
$$

where $g=0,1,2$. The decision result of each target consumer is described by comparing $X_{g}(i, t)$ with probability threshold defined in Section 4.3.3.

\subsubsection{Network Topology Generation and Purchase} Probability. The link distribution of a real social network is scale-free and small-world. To generate an environment similar to a real social network, the algorithm from the literature is adopted [1], and it adds two modification rules based on the preferred connection algorithm [38-40]. First, a cliff-like structure is created, in which the newly added agent is connected to a random subset of $50 \%$ of the central agent (i.e., the friend of a friend) after first connecting with the larger central agent. Second, the small-world feature is added. This increases connections between all unconnected agents by $10 \%$ (similar to weak connections in the real world).

Based on Han et al. [41], according to the network topology structure of the above consumers, the probability that potential consumers with the degree of $k$ at time $t$ have $n$ buying consumer neighbors is 


$$
\Theta(t)=C_{k}^{n} \psi^{n}(t)\{1-\psi(t)\}^{k-n}=\frac{k !}{n !(k-n) !} \psi^{n}(t)\{1-\psi(t)\}^{k-n}
$$

where $\psi(t)$ represents the probability that potential consumers with degree $k$ at time $t$ are connected with purchasing consumers with degree $k^{\prime}$. The expression is as follows:

$$
\psi(t)=\frac{\sum_{k^{\prime}} k p_{B}\left(k^{\prime}\right) F_{k^{\prime}}^{B}(t)}{\sum_{k^{\prime}} k p_{B}\left(k^{\prime}\right)}
$$

where $k$ is the degree of potential consumers, $k^{\prime}$ is the degree of purchasing consumers among the neighbors of potential consumers, $p_{B}\left(k^{\prime}\right)$ is the proportion of the number of purchasing consumers with degree $k^{\prime}$ at time $t$ among the total number of purchasing consumers, and $F_{k^{\prime}}^{B}(t)$ is the proportion of the number of purchasing consumers with degree $k^{\prime}$ at time $t$ among the number of all consumers.

Consumers may be more likely to trust certain network members in the formed network structure. In this paper, the interaction degree parameter $\delta_{i}$ is used for presentation. The higher the degree of interaction, the higher the trust among members of the consumer network. At the same time, consumers have conformity psychology, which is reflected by the conformity degree parameter $\xi_{i}$. The probability that potential consumer $i$ transforms into purchasing consumer under the condition of consumer heterogeneity caused by network topology is $\Theta(t) \xi_{i} \delta_{i}$.

\subsubsection{Consumer Heterogeneity Resulting From Information} Flows and Purchase Probability. In the process of purchasing new products, potential consumers are in a wait-and-see state due to their lack of in-depth understanding of new product information. They change their own purchasing attitude and behavior by constantly learning and collecting information. It is assumed that potential consumers make a purchase decision based on two product attributes: performance and price. Usually, consumers know the price, but are uncertain about the performance of new products. Potential consumers update their cognition of uncertainty about new product performance according to the amount of information received, and individual potential consumers have heterogeneity in the initial cognition and perceived reliability of information. According to Chatterjee and Eliashberg [20], all potential consumers are assumed to be risk-averse in their purchase decisions of new products. At the same time, the degree of risk aversion for each consumer is different, and there is a linear relationship between the utility of consumers and price. Researchers adopt the utility function in the form of an exponential [13, 42]. Each information unit is assumed to contain only one piece of information, and utility function $U_{j}\left(\omega_{\mathrm{ja}}, p_{\mathrm{jt}}\right)$ under the two attributes of the new product price and performance can be obtained as follows:

$$
U_{j}\left(\Theta_{j \beta}, p_{j t}\right)=1-\exp \left(\eta_{j} \Theta_{j \beta}\right)-\kappa p_{j \mathrm{t}}
$$

where $\omega_{j \beta}$ refers to the uncertain cognition of potential consumer $j$ on the performance of the new product after receiving the $\beta$ th information unit, and $\omega_{j \beta} \sim N\left(\theta_{j \beta}, \vartheta_{j \beta}^{2}\right)$. $\theta_{j \beta}$ refers to the expectation of the uncertain cognition of potential consumer $j$ on the new product's performance after receiving the $\beta$ th information unit. $\vartheta_{j \beta}^{2}$ refers to the variance of the uncertain cognition of potential consumer $j$ on the new product's performance after receiving the $\beta$ th information unit. $\eta_{j}$ is the risk aversion degree of potential consumer $j, \kappa$ is the relative importance of the new product price, and $p_{\mathrm{jt}}$ is the price of the new product. The estimated value of parameter $\eta_{j}$ is $\widehat{\eta}_{j}=-\eta^{*} /[\ln (1-C / 10)]$, where $\eta^{*}$ is the normal number referring to the number of problems faced by consumers when deciding whether to buy. $C$ is the number of choices in $\eta^{*}$.

To update parameters $\theta_{j \beta}$ and $\vartheta_{j \beta}^{2}$ using the Bayes rule [43], the formulas are expressed as follows:

$$
\begin{aligned}
& \theta_{j \beta}=\frac{\left(\theta_{j \beta-1} / \vartheta_{j \beta-1}^{2}+v_{\beta} / \sigma^{2}\right)}{\left(1 / \vartheta_{j \beta-1}^{2}+1 / \sigma^{2}\right)}, \\
& \vartheta_{j \beta}^{2}=\frac{1}{\left(1 / \vartheta_{j \beta-1}^{2}+1 / \sigma^{2}\right)},
\end{aligned}
$$

where $v_{\beta}$ is the performance level of the new product conveyed by the $\beta$ th information unit, and $\nu_{\beta} \sim N\left(\mu, \sigma^{2}\right) . \mu$ is the true performance level of the new product. $\sigma^{2}$ reflects the volatility of the performance level of the new product conveyed by the information unit.

According to Chatterjee and Eliashberg [20], $\Gamma_{j}$ represents the impact of the information before the new product enters the market on the initial cognition of potential consumer $j$, and $\Psi_{j}$ represents the price hurdle to the purchase decision of potential consumer $j$. Thus, $\Gamma_{j}$ and $\Psi_{j}$ are expressed as follows:

$$
\begin{aligned}
\Gamma_{j} & =\left[\frac{\eta_{j} \vartheta_{j 0}^{2}}{2}-\left(\frac{1}{\eta_{j}}\right) \ln \left(1-\kappa p_{\mathrm{jt}}\right)-\theta_{j 0}\right]\left[\frac{\sigma^{2}}{\vartheta_{j 0}^{2}}\right], \\
\Psi_{j} & =-\left(\frac{1}{\eta_{j}}\right) \ln \left(1-\kappa p_{\mathrm{jt}}\right) .
\end{aligned}
$$

Finally, according to Cox and Miller et al. [44], the probability of potential consumers purchasing new products under the consumer heterogeneity caused by information flow is

$$
\begin{aligned}
P\left(t \mid \Gamma_{j}, \Psi_{j}\right)= & \Phi\left(\frac{-\Gamma_{j}+\left(\mu-\Psi_{j}\right) \mathrm{H}(t)}{\sigma \sqrt{\mathrm{H}(t)}}\right) \\
& +\exp \left[\frac{2 \Gamma_{j}\left(\mu-\Psi_{j}\right)}{\sigma^{2}}\right] \Phi\left(\frac{-\Gamma_{j}-\left(\mu-\Psi_{j}\right) \mathrm{H}(t)}{\sigma \sqrt{\mathrm{H}(t)}}\right),
\end{aligned}
$$

where $\Phi(\bullet)$ indicates that the random variable obeys the standard normal distribution. $\mathrm{H}(t)=\sum_{t=0}^{t} h(t) W$, where $h(t)$ is the information rate at time $t, W$ is the total number of 
new product information, and $\mathrm{H}(t)$ is the cumulative amount of information collected by potential consumers in time $t$.

\section{A Pilot Study of Short Life Cycle Experience Products}

This section describes the preliminary studies carried out in an experimental environment. It utilizes the real situation of films, which are considered as short life cycle experience products, for the experiment. Accordingly, this study summarizes the key parameters used in the above model. Moreover, it estimates the specific parameters of the model's individual level, network topology, and information from the collected data. Finally, scenarios for evaluating and implementing various strategy choices are presented.

4.1. Key Parameters and Data Collection. Early studies, such as by Zhang and Zhang [36], generally assumed parameter values due to lack of actual data. However, this could not reflect the real situation of new products in the market. Therefore, this research collects real historical data of films to determine the parameters to better reflect the real Chinese film market. The parameter values and their settings are shown in Table 2. The sources and methods for obtaining these parameters are described in depth below.

The parameters in Table 2 can be divided into three categories. The first category involves parameters describing consumers' characteristics. Consumers' descriptions of price and quality sensitivity are, respectively, $\hbar_{i}$ and $\lambda_{i}$, and the setting method of these two parameters is based on the literature [18]. Sensitivity $\varepsilon_{\text {it }}$ of consumers to advertisements is also obtained from the literature [31]. Consumers' sensitivity to time $\varphi_{\text {it }}$ is obtained by calculating the estimated results in Song and Chintagunta [13].

The second category involves the properties of film products. These include price $p_{\mathrm{it}}$ and $p_{\mathrm{jt}}$, its average $\widetilde{p}$, advertising intensity $L_{t}$, average quality $\widetilde{Q}$, rating $p_{f}$, studio attraction $z_{x}$, and time parameter $d_{t}$. By collecting data on 183 films with a domestic box office collection of more than 1 million yuan from 2017 to 2019, missing values were removed, and the data on 138 eligible films were selected. The above parameters are estimated and analyzed using statistical data. Price data are collected from the website of the Maoyan professional edition; box office and studio attraction data come from China's box office website; scoring data are collected from the Douban website. Advertising intensity is defined as the length of the broadcast time for an advertisement, and the data value is set as in Cui and Cheng [45]. The time parameter is defined as a 01 variable, the working days are 0 , and the weekends are 1 .

The third category is information flow-related features. The remaining parameters in Table 2 measure the stability, reliability, and amount of information of the information flow. The definitions of these parameters are consistent with those in the model in Sections 3.3.3 and 4.2. The amount of information comes from the official Weibo accounts of 138 films. Data on information flow stability and reliability can be referred to from Chatterjee and Eliashberg [20] for the measurement and estimation of parameters.

4.2. Data Processing and Initialization. In this study, Microsoft Office Excel, SPSS, and MATLAB were used to analyze the data. The few missing data items on price are filled using the mean value method based on the adjacent points in SPSS. The daily average price distribution curve of the 138 films is shown in Figure 3.

Film quality consists of two parts: rating and studio appeal [46]. Studio appeal is defined as the sum of the topthree film box office hits among all films, including analytical films. Based on the regression analysis of 138 films' data in MS Excel, the definition of film quality is reasonable and applicable. The regression expression is $\log _{10} Q=0.67 p_{f}+0.19 \log _{10} z_{x}$. Results of the regression analysis are shown in Table 3 . The curve fitting was performed using MATLAB Cftool (curve fitting tool) based on the least squares method to determine the functional relationship between the film information rate $h(t)$ and time. It is concluded that about $50 \%$ of the films obey a Gaussian distribution, and the functional relationship is expressed as $h(t)=a_{1} * \exp \left\{-\left[\left(t-b_{1}\right) / c_{1}\right]^{2}\right\}$. The value ranges of $a_{1}, b_{1}$, and $c_{1}$ are shown in Table 2 .

After receiving the information flow, the estimated values of the uncertain cognitive parameters $\theta_{j \beta}$ and $\vartheta_{j \beta}^{2}$ for the performance of new products are as follows:

$$
\widehat{\theta}_{j \beta}=\frac{\sum_{l=1}^{21} L_{j \beta l} x_{l}}{\sum_{l=1}^{21} L_{j \beta l}} \text { and } \widehat{\vartheta}_{j \beta}^{2}=\frac{\sum_{l=1}^{21} L_{j \beta l}\left(x_{l}-\widehat{\theta}_{j \beta}\right)^{2}}{\sum_{l=1}^{21} L_{j \beta l}} \text {. }
$$

According to Chatterjee and Eliashberg [20], assuming that the performance level of the new product ranges from 0 to 100 and is divided into 21 intervals based on $5, x_{l}$ represents the score value at 21 points, namely, $x_{l}=5(l-1) . l$ stands for 21 interval endpoints. $L_{j \beta l}$ is the relative likelihood score, ranging from 0 to 100 . The setting of the parameter values is shown in Table 2 .

4.3. Sample Scenarios. Simulations allow various scenarios to play out for assessing strategic choices. In Section 5, we provide illustrative results for an example scenario. The following describes the scenarios used.

4.3.1. Network Topology. According to the specific social connection and communication frequency of film products, a scale-free network model based on preference connection generation is improved. The model comparison between scalefree network-degree distribution (Figure 4(a)) and network topology degree distribution in this study (Figure 4(b)) is shown in Figure 4 when the average degree $\widetilde{k}$ of the improved network model in this study is 3 , it shows the same heavy-tailed distribution as the scale-free network, with the same slope value and a small intercept error, indicating that the scale-free network is a special case of the model in this study. Furthermore, 
TABLE 2: Initial parameter settings of the basic parameters.

\begin{tabular}{|c|c|c|c|c|c|}
\hline Parameter & Scale & Distribution & Parameter & Scale & Distribution \\
\hline$t$ & $1-60$ & Integers within the interval & $\varphi_{\text {it }}$ & $0<\varphi_{\text {it }}<1$ & $\begin{array}{l}\text { Normal distribution } \\
\quad N(0.587,0.03)\end{array}$ \\
\hline$\hbar_{i}$ & $\hbar_{i}>1$ & Normal distribution $N(3.6,1.6)$ & $d_{t}$ & $\begin{array}{c}0 \text { or } 1 \\
a_{1}:[0.04,6.59]\end{array}$ & Constant \\
\hline$\lambda_{i}$ & $0<\lambda_{i}<1$ & Normal distribution $N(0.6,0.2)$ & $h(t)$ & $\begin{array}{l}b_{1}:[-60.66,7.86] \\
c_{1}:[0.51,31.71]\end{array}$ & Random distribution \\
\hline$p_{\mathrm{it}}, p_{\mathrm{jt}}$ & $0-400$ & Normal distribution $N(34.5,93.19)$ & $W$ & {$[0,1000]$} & Random integer in the interval \\
\hline$\varepsilon_{i t}$ & $1-5$ & Normal distribution $N(2.77,0.69)$ & $\mu$ & $0<\mu<100$ & Random integer in the interval \\
\hline$\tilde{\tilde{p}}$ & 34.5 & Constant & $\eta_{j}$ & $(0,+\infty)$ & Random distribution \\
\hline$\widetilde{Q}$ & $45,852.79$ & Constant & $\kappa$ & {$[0,0.02)$} & Uniform distribution \\
\hline$L_{t}$ & $(0,60)$ & $\begin{array}{c}\text { Random distribution of integers within } \\
\text { the interval }\end{array}$ & $l$ & {$[1,21]$} & Integers within the interval \\
\hline$p_{f}$ & $1-10$ & Integers within the interval & $L_{j \beta l}$ & $0-100$ & $\begin{array}{c}\text { Random integer in units of } 5 \text { in an } \\
\text { interval }\end{array}$ \\
\hline$z_{x}$ & $\begin{array}{c}1,007.3-1,605 \\
163\end{array}$ & Random distribution & $x_{l}$ & $0-100$ & $\begin{array}{c}\text { Random integers and multiples } \\
\text { of } 5\end{array}$ \\
\hline
\end{tabular}

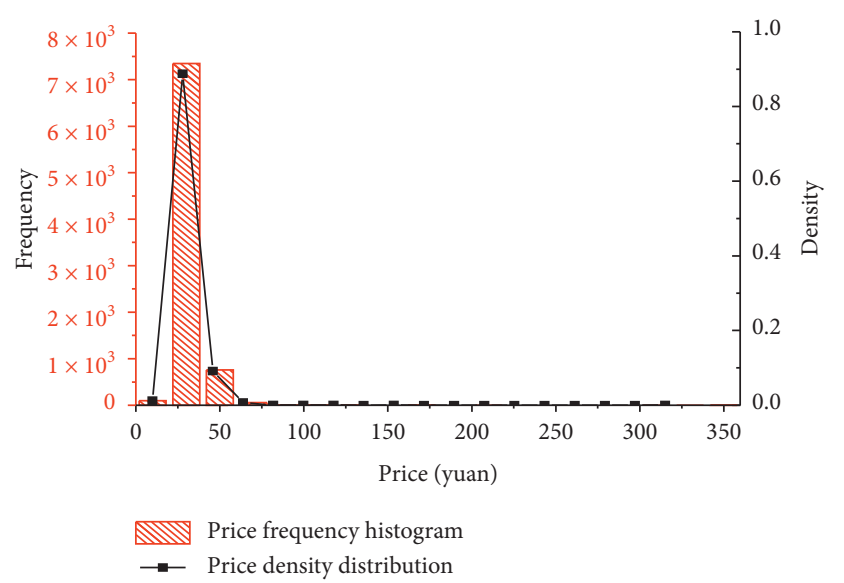

FIgURE 3: The price frequency histogram and price density distribution.

TABLE 3: Linear regression analysis results on film quality.

\begin{tabular}{lccc}
\hline \multicolumn{4}{c}{ Regression statistics } \\
\hline Multiple $R$ & 0.992781 & Adjusted $R$ square & 0.97821 \\
$R$ Square & 0.985615 & Standard error & 0.498577 \\
\hline
\end{tabular}

the model presented in this study can better reflect the real film consumer network. Real network topology will be different due to differences in the market environment and the characteristics of the sales object. In this study, average degree $\tilde{k}$ of the network topology is set to the five measurement levels of $4,6,8,10$, and 12. The test is run in the NetLogo 6.1 .0 environment. Figure 5 depicts the network-degree distribution instance created with $\widetilde{k}$ for the sets of 6 and 8. It shows a heavy-tailed distribution. Unlike the typical scale-free network, it shows scale-free characteristics after the inflection point and reaches a peak at the mean degree. This phenomenon shows that, in reality, due to the number of nodes distributed around the average degree and the occurrence of heavy-tailed area, most people have a certain number of friends.
4.3.2. Price and Quality. This study estimates the average price of films and tests five average price levels of films to represent different pricing strategies. Moreover, film quality is divided into five average levels.

\subsubsection{Transition Probability Threshold Distribution.} According to Lin et al. [19], the probability distribution is used to describe the decision change threshold of target consumers. The probability thresholds of a single target consumer turning into a potential consumer, a purchasing consumer, and an abandoning consumer are represented by $\mathrm{X}_{0}^{*}(i, t), \mathrm{X}_{1}^{*}(i, t)$, and $\mathrm{X}_{2}^{*}(i, t)$, respectively. The probability thresholds of potential consumers turning into buying consumers and abandoning consumers are represented by $\mathrm{X}_{3}^{*}(j, t)$ and $\mathrm{X}_{4}^{*}(j, t)$, respectively.

Definition 1. According to the principle of a high probability event and average distribution, the threshold of transition probability of target consumers to other types of consumers is subject to $\mathrm{X}_{g}^{*}(i, t) \sim \operatorname{Uniform}(m, n), g=0,1,2$, and the probability distribution of the three transition thresholds is as follows: $\mathrm{X}_{1}^{*}(i, t) \sim \operatorname{Uniform}(0.8,1), \quad \mathrm{X}_{0}^{*}(i, t) \sim$ Uniform $(0.3,0.8)$, and $\mathrm{X}_{2}^{*}(i, t) \sim \operatorname{Uniform}(0,0.3)$.

Definition 2. Similarly, the threshold probability distributions of potential consumers' transition to purchasing consumers and abandoning consumers are $\mathrm{X}_{3}^{*}(j, t) \sim$ Uniform $(0.5,1)$ and $\mathrm{X}_{4}^{*}(j, t) \sim \operatorname{Uniform}(0,0.5)$, respectively.

The threshold rationality and robustness defined above will be illustrated by the sensitivity analysis in Section 6 .

4.3.4. Degree of Conformity and Interaction Heterogeneity. In the model, conformity and interaction heterogeneity are represented by $\xi_{j}$ and $\delta_{j}$, respectively. Consumers are often influenced by the majority of people and follow the thoughts or behaviors of masses. Reinstein and Snyder [47] define the conformity effect as the behavior of decision makers following the decisions of predecessors. In the era of Web 2.0, 


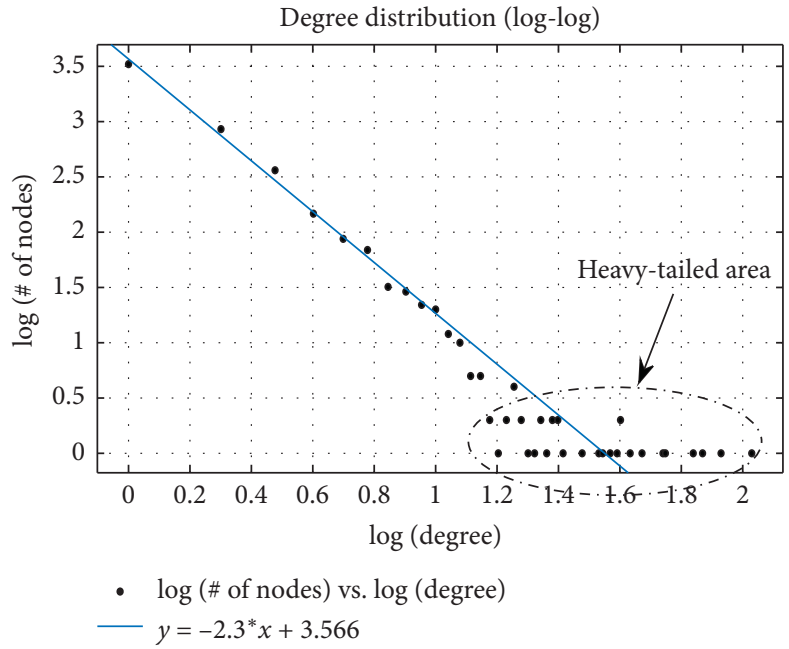

(a)

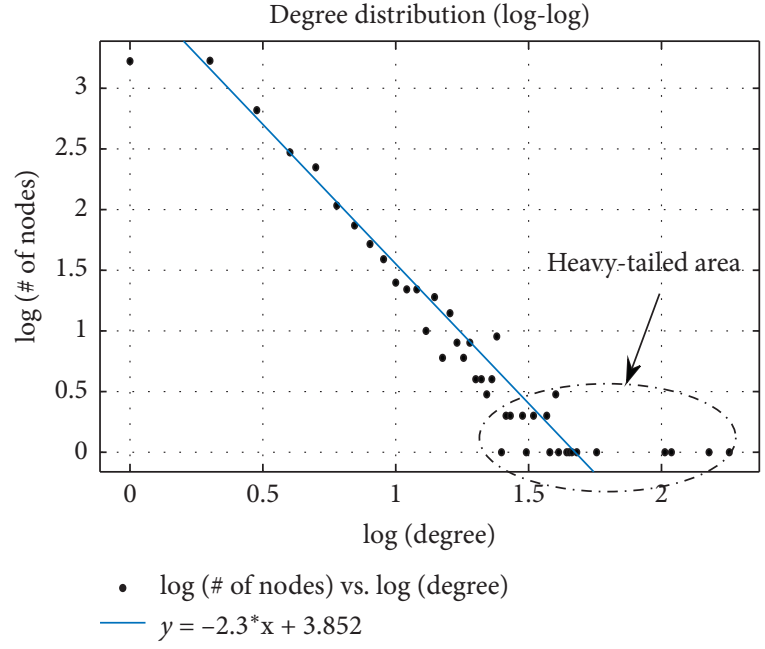

(b)

FIgUre 4: Comparison diagram of scale-free network-degree distribution and network topology degree distribution in this study. (a) Scalefree network-degree distribution. (b) Network topology degree distribution in the study.

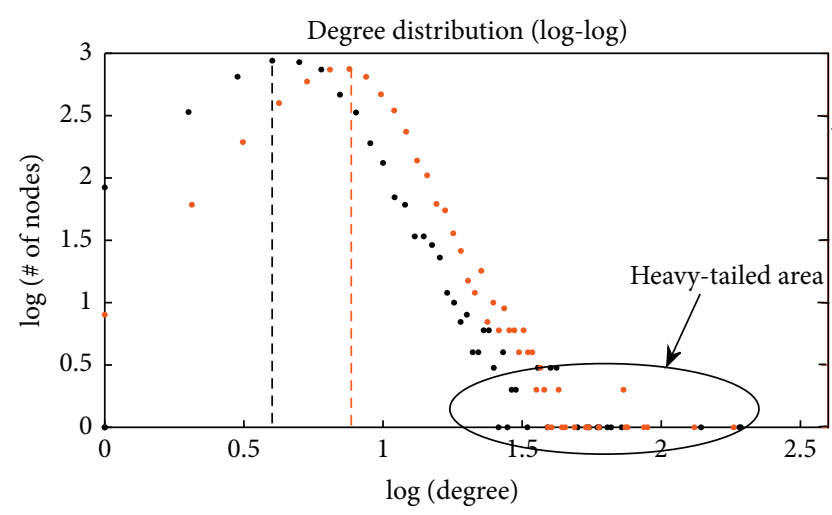

- The average degree of 6

- The average degree of 8

FIGURE 5: An example of the distribution of network topologies.

online interactions among consumers have become an indispensable social communication activity for people. Coupled with offline communication, it has a profound impact on consumer psychology and purchasing behavior. However, there are differences in consumer conformity and interaction. In the simulation experiment, the two types of heterogeneity were divided equally into 20 intervals between 0 and 1 to analyze the influence of conformity and interaction heterogeneity on film diffusion.

\section{Simulation Results}

This section provides illustrative results for a sample scenario. These are results of a preliminary test of the model's impact at the individual level (consumer characteristics and behavior) on the overall level (final market share and rate of diffusion). The simulation is performed in NetLogo 6.1.0, and the simulation process is shown in Figure 6. In Figure 6, the solid arrows represent the traversal process inside a loop, and the dotted arrows represent the judgment process before entering the next loop. For each possible scenario, different behavior combination results are realized through the behavior space in this study. Films are short life cycle products, and statistical historical data show that a film's box office collection hardly changes in 60 days. Therefore, the simulation time $t$ was set to 60 days in this study.

In Sections 5.1 and 5.2, the effects of the degree of conformity heterogeneity and interaction heterogeneity based on different network topologies on the film diffusion scale and speed are provided. To illustrate the influence of price and quality changes on film diffusion scale and speed, the simulation and analysis results are presented in Section 5.3.

5.1. Conformity Heterogeneity and Network Topology. This section focuses on the influence of conformity heterogeneity and consumer heterogeneity caused by network topology on the film product diffusion scale and speed. The scale of film diffusion is measured by market share, which is defined as the proportion of ticket purchasers in the total population during film release. The average speed of film product diffusion is expressed as the number of people buying tickets per unit time.

5.1.1. Effect of Conformity Heterogeneity and Network Topology on the Diffusion Scope. The degree of conformity heterogeneity reflects the difference in conformity heterogeneity in the consumer population, and its value range is divided equally into 20 cells with an interval of 0 to 1 . Here, 0 indicates that all consumers are homogeneous, and 1 indicates the largest difference in heterogeneity. The network with different average degrees reflects that the network topology has a different degree 


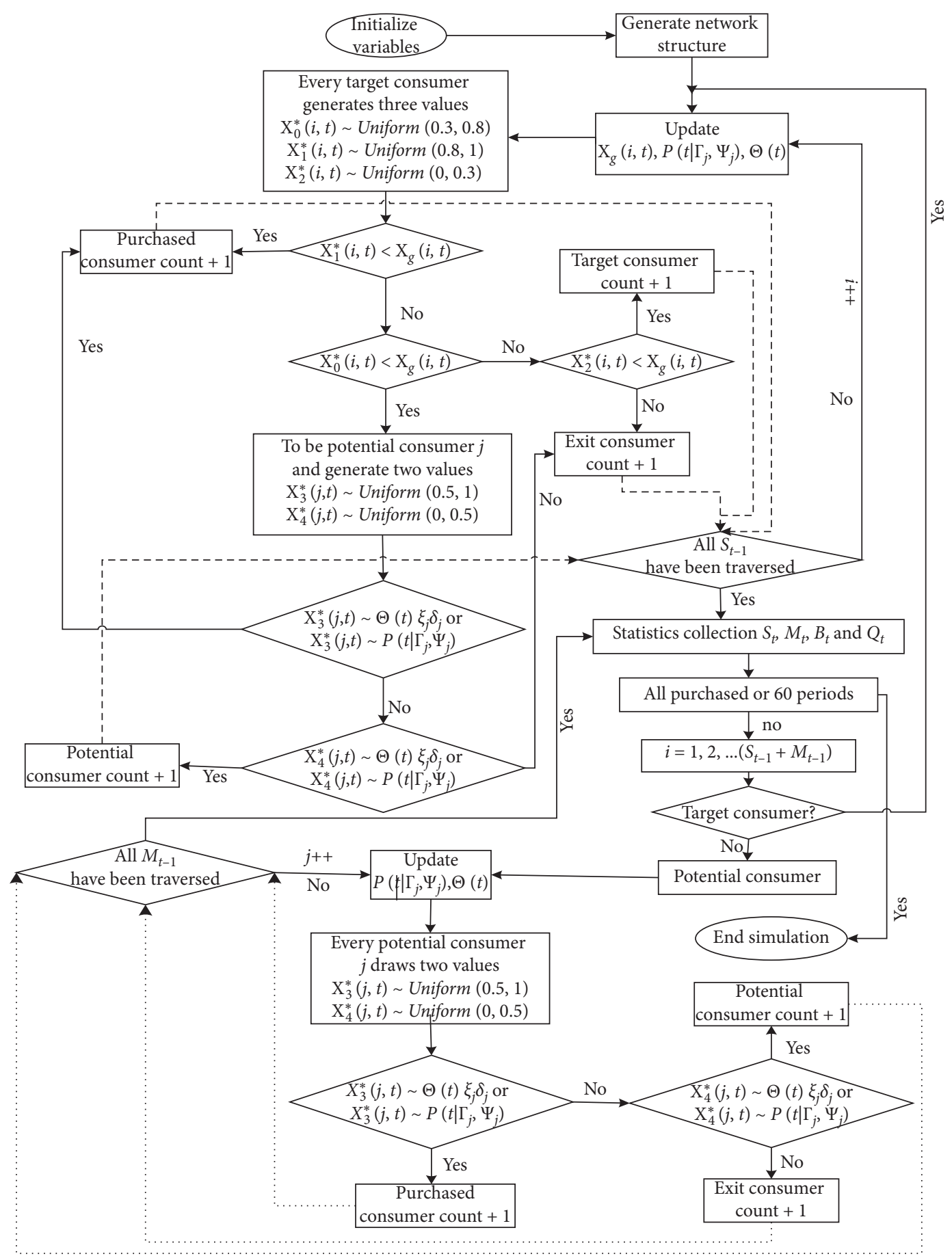

Figure 6: Overview of the simulation process under the coupling model.

distribution, which represents the local structural characteristics of the network.

Figure 7 describes the changes in the final market share with the degree of conformity heterogeneity under three network topologies $(\widetilde{k}=6 / 8 / 10)$. It can be observed from Figure 7 that when $\widetilde{k}=6$, with the increase of conformity heterogeneity degree, the number of maximum values with obvious gap in market share is the largest. Therefore, compared with $\widetilde{k}=8$ or $\widetilde{k}=10$, when there are fewer neighbor nodes, it is easier for consumers to appear the maximum market share with the increase of conformity heterogeneity degree. As shown in Figure 7, under the network structure with $\widetilde{k}=6$, when the degree of conformity heterogeneity reaches the maximum value of 1 , the market share reaches the maximum value. However, the network market share with $\widetilde{k}=8$ or $\widetilde{k}=10$ is relatively low. This shows that, in reality, when there are relatively more neighbors of consumers, it is easier for consumers to exhibit an uncertain psychological state, which is not conducive to making purchase behaviors. 


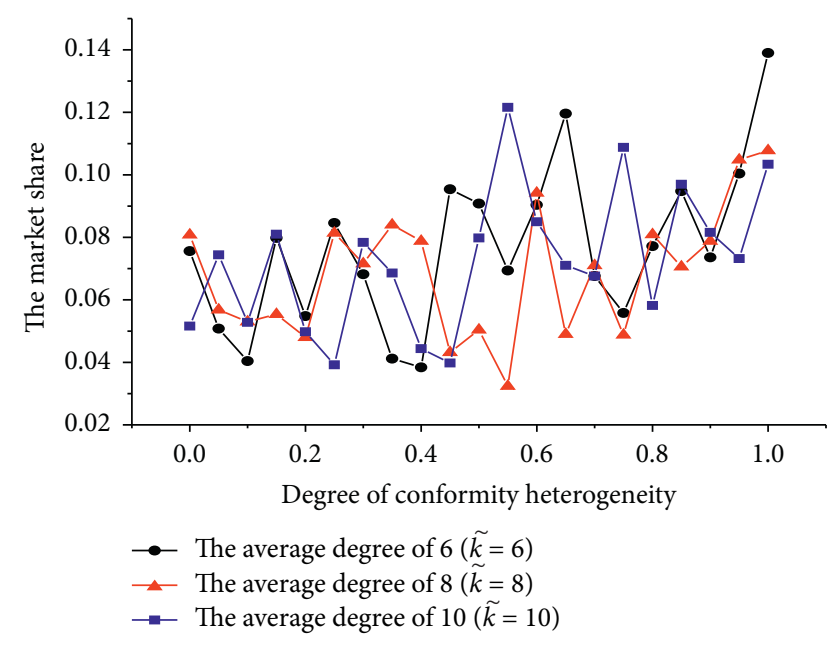

Figure 7: Effects of conformity heterogeneity on the diffusion scale based on different network topologies.

Compared with the market share at the zero point of homogeneity, the market share fluctuates greatly under different degrees of conformity heterogeneity, and the overall trend is toward growth. Therefore, it can be concluded that when the degree of consumer conformity is relatively large in the film diffusion process, the effect on the diffusion scale is more significant. It can also be observed from Figure 7 that when the average degree is 8 , the market share fluctuation is relatively stable with the increase of conformity heterogeneity degree. This indicates that the influence of conformity heterogeneity on market share at $\widetilde{k}=8$ is relatively weak compared with that at $\widetilde{k}=6$ or $\widetilde{k}=10$.

5.1.2. Effect of Conformity Heterogeneity and Network Topology on the Diffusion Rate. The degree of consumer conformity heterogeneity has an impact on not only the scale of film diffusion but also its average speed, as shown in Figure 8. In MATLAB R2017b, the spline interpolation function is used to display the dynamic change process of scatter points. It can be observed from the figure that, for a network with average degrees of 6 and 8, the degree of conformity heterogeneity also has the greatest impact on the average diffusion speed when the value is 1 , and the average diffusion speed reaches the maximum. In Section 5.1.1, when the average degree is 8 , the fluctuation of market share is stable, but the effect on the average diffusion speed easily appears to be minimum. This indicates that, in a network with an average degree of 8 , for consumers with different degrees of conformity, although the impact on the diffusion scale is small, the impact on the diffusion speed is more significant. At the same time, when the average degrees are 6 and 10 , the curve fluctuation is very similar, and the difference in the degree of conformity heterogeneity at the extreme value is 0.01 . This indicates that the effect of conformity heterogeneity on diffusion speed is similar in the two network environments, and the difference is small.

In a network environment with an average degree of 8 , the effect of conformity heterogeneity on diffusion velocity is almost symmetrical, with a symmetry point of 0.5 . A possible explanation is that there is a threshold for the degree of consumer conformity heterogeneity, and the change after exceeding the threshold is similar to that before the threshold. That is, the influence of conformity on the average diffusion speed is controlled by the threshold value. This shows that the degree of conformity heterogeneity only has a threshold in a specific network topology.

\subsection{Interaction Heterogeneity and Topological Structure}

5.2.1. Effect of Interaction Heterogeneity and Topological Structure on the Diffusion Scope. Every consumer's behaviors and habits before watching films are not the same: some prefer face-to-face communication to discuss whether a film is worth watching; some like to search for relevant information on web pages, such as comments or forecasts; and some like to use social media to communicate. The number and frequency of consumer communication are different, which indicates that the degree of consumer interaction heterogeneity is different. Similarly, 0 means that consumers are homogeneous, and 1 means that the degree of interaction heterogeneity varies the most.

As shown in Figure 9, for three network topologies $(\widetilde{k}=6 / 8 / 10)$, as the degree of interaction heterogeneity increases, the market share tends to be maximized near both ends and the middle position. The overall fluctuation of market share is similar to a " $W$ " shape. One possible explanation is that the interaction between consumers is skillbased, with zero communication, deep communication, or just a little more, in which case it is easy to achieve optimal market share. In addition, in Figure 9, in different network topologies, the degree of consumer interaction heterogeneity influences the final market share of films. As shown in the figure, when the degree of interaction heterogeneity is 1 , the market share of films in different network environments reaches a maximum. This indicates that regardless of whether the number of consumers' neighbors is large or small, the interaction heterogeneity has a significant effect on the diffusion scale.

\subsubsection{Effect of Interaction Heterogeneity and Topological} Structure on the Diffusion Rate. The results of the simulation experiment are calculated and processed, and the spline interpolation function is used in MATLAB R2017b to show the dynamic change process of scattered points. As shown in Figure 10, under different network topologies, the influence of consumer interaction heterogeneity on the average diffusion speed for films is explained.

Figure 10 shows that when the degree of interaction heterogeneity is 1 , it has the greatest impact on the average diffusion speed of films, which is the same as the impact on the diffusion scale. According to the figure, the variation in the three curves is almost symmetrical on both sides of axis 0.5 . A possible explanation is that there is a threshold for the degree of consumer interaction heterogeneity, and postthreshold changes are similar to those before the threshold. That is, the influence of the interaction frequency on the 


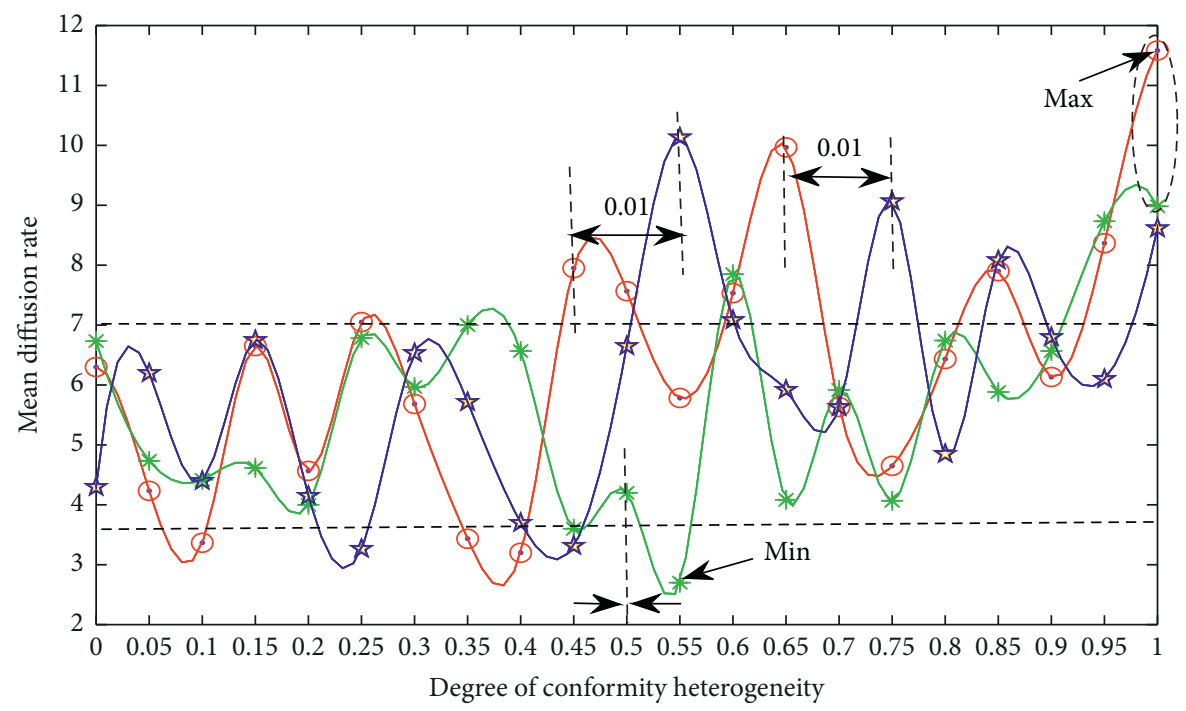

The average degree of 6
The average degree of 8
The average degree of 10

FIGURE 8: Influence of conformity heterogeneity on mean diffusion rate based on different network topologies.

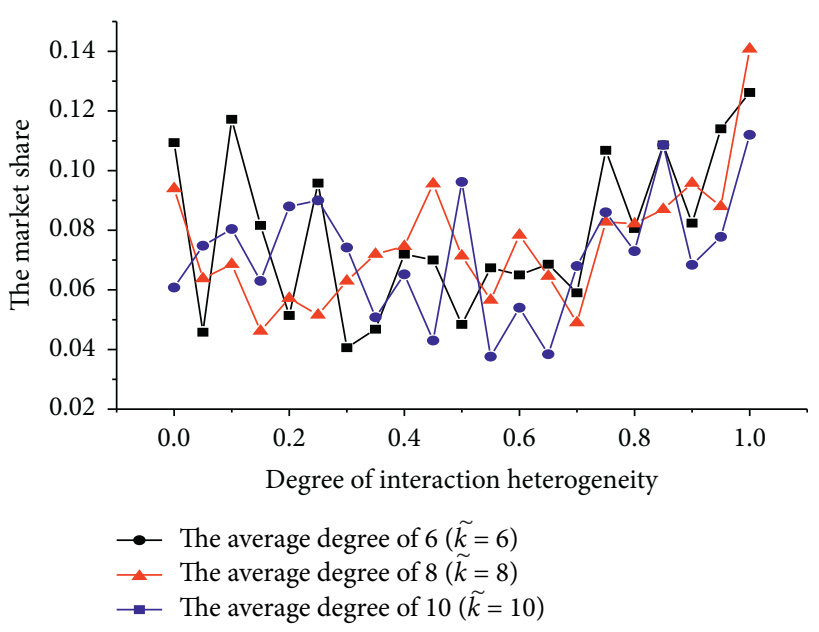

FIGURE 9: Influence of interaction heterogeneity on the diffusion scale based on different network topologies.

average diffusion speed is controlled by the threshold. Figure 10 shows that the threshold value is 0.5 . This shows that the degree of interaction heterogeneity has a threshold in a given network topology.

5.3. New Product Quality, Price, and Network Topology. The quality of new products determines consumer satisfaction, while the price affects the profits of enterprises. According to formulas (6) and (7), quality and price determine consumers' sensitivity to quality and price, respectively. If the price of high-quality new products is too high or too low, product diffusion may fail, and vice versa. A reasonable marketing mix strategy plays a key role in the success of new product diffusion. This section discusses the impact of the combination of quality and price changes on the scale and speed of new product diffusion under different network topologies.

5.3.1. Effect of Quality, Price, and Network Topology on Diffusion Scope. This section divides the average quality, price, and network average into five levels. According to the parameter setting in Section 4.1 and data processing in Section 4.2 , the average quality and price are $45,852.79$, and 34.5 yuan, respectively. Based on this, we set five levels, which fluctuate in units of 100 million yuan and 10 yuan. The impact of changes in average quality and price on the films' market share under different network topologies is shown in Figure 11.

Figure 11 presents a map of a 3D spatial mapping surface with projection, in which the color scale is marked according to the size of the market share. When the average degree of the network is $6,8,10$, and 12 , respectively, regions with a large market share appear in regions with high quality and high price, low quality and low price, and some regions with medium quality and low price. This indirectly indicates that the model can reflect reality. In a network environment with an average degree of 8 , the probability of film diffusion success is relatively large.

5.3.2. Effect of Quality, Price, and Network Topology on Diffusion Rate. According to Section 5.3.1, the network diffusion success rate with an average degree of 8 reaches the maximum. To facilitate the analysis, the influence of the change in average quality and price on the average diffusion rate was discussed only when the average degree is 8 ; see Figure 12. The color scale was determined according to the size of the average diffusion rate. Other network topology situations were analyzed similarly. 


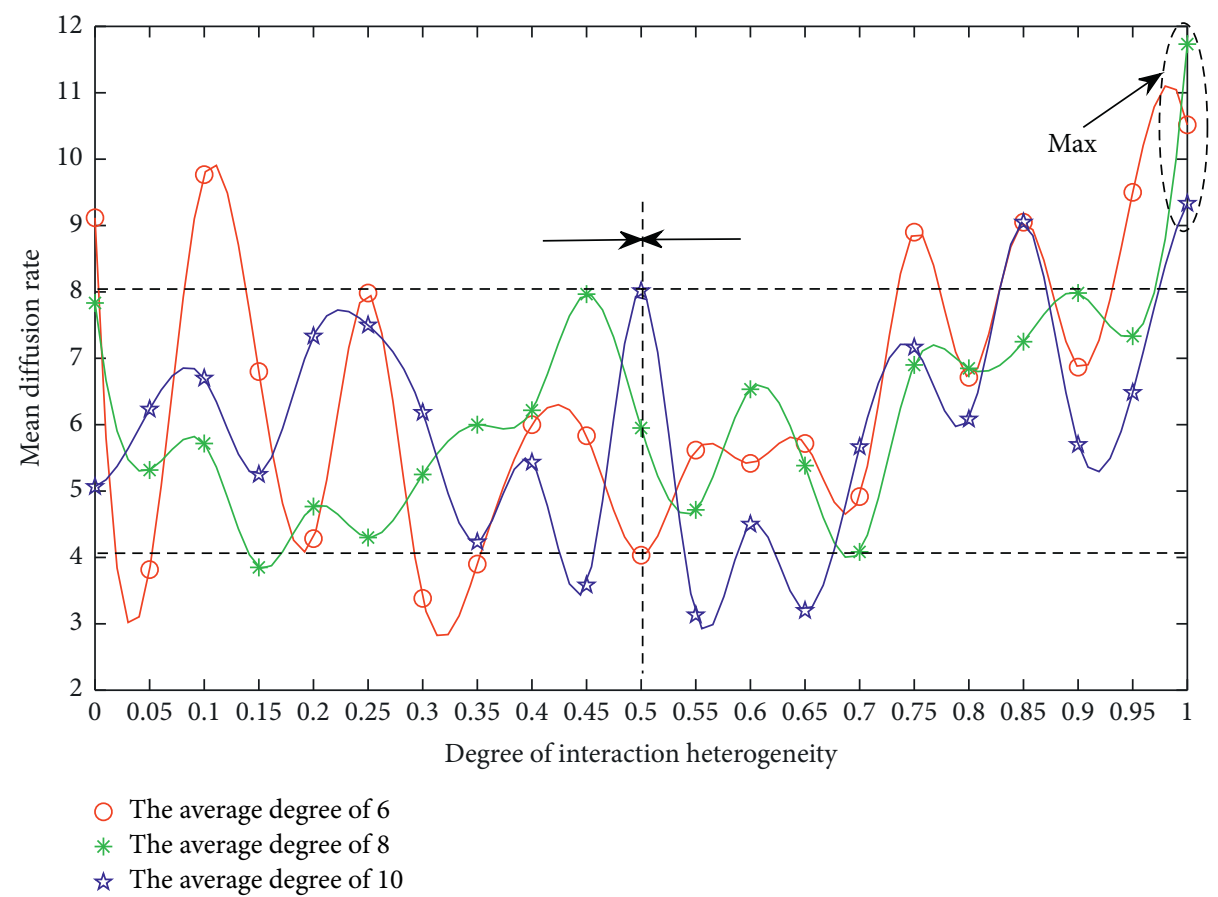

FIGURE 10: Influence of interaction heterogeneity on mean diffusion rate based on different network topologies.

In Figure 12, the area with the faster average film diffusion rate is located in the arrow area at the top right of the figure and the dashed line with a horizontal coordinate of 34.5 yuan, which shows that films with medium to high prices are more likely to have successful diffusion under situations of medium and high quality. Regarding the price of medium-quality films, there is a phenomenon of polarization, which indicates that there is no fixed pricing standard for medium-quality products worthy of reference, and there is strong uncertainty that is prone to extreme situations. Meanwhile, the average price of 34.5 yuan is a standard pricing reference for any quality range. In Figure 12, there are some special abnormal values, such as low quality and high price, while the average diffusion speed is large. A possible explanation is that the marketing propaganda is strong, and the diffusion is fast when word of mouth is not involved in the early stage.

\section{Sensitivity Analysis and Validation}

Regarding sensitivity, as a method to evaluate the robustness of the results of this study, we performed a sensitivity analysis on population size. Experiments with different population sizes of consumer agents $(1,000,5,000,10,000$, and 15,000$)$ show that the film market share of a population size of 5,000 or more is very stable over time, as shown in Figure 13.

At the same time, we conducted a sensitivity test on the probability distribution thresholds in Section 4.3.3. The changes of the five probability thresholds are shown in Table 4 . The remaining parameter values in the system are consistent, as shown in Table 2. Two of the five probability thresholds are changed each time. In NetLogo 6.1.0, we simulate each group of parameters and get the number of purchase consumers changing with time under each group probability distribution threshold. Furthermore, the deviation rates of the six comparison groups and the reference group were calculated separately, and the variation of each group's deviation rate with time was analyzed to obtain the sensitivity analysis of the probability distribution threshold, as shown in Figure 14. It can be seen from Figure 14 that the deviation rate between the six comparison groups and the reference group gradually stabilizes near zero over time, indicating that the change of the threshold has little impact on the total number of consumers, the system has weak sensitivity to the threshold, and the simulation results have little risk of deviation from reality and strong robustness.

Moreover, validating the model in this study is a challenging task. In the context of innovation diffusion, difficulties arise from creating realistic networks, collecting data at the individual level, and tracking diffusion at the nonaggregate level [48]. In this study, a large number of tests are conducted to check the conceptual validity, internal validity, external validity at the microlevel, external validity at the macrolevel, and cross-model validity. When the conceptual model fully describes the phenomena in the real world, conceptual validity is reached; internal validity refers to the correct implementation of computer code; external validity involves the relationship between model behavior and the actual system; and cross-model validation compares the results of different modeling approaches.

The conceptual validity of the model is based on the solid foundation in mature theories, especially the new product 


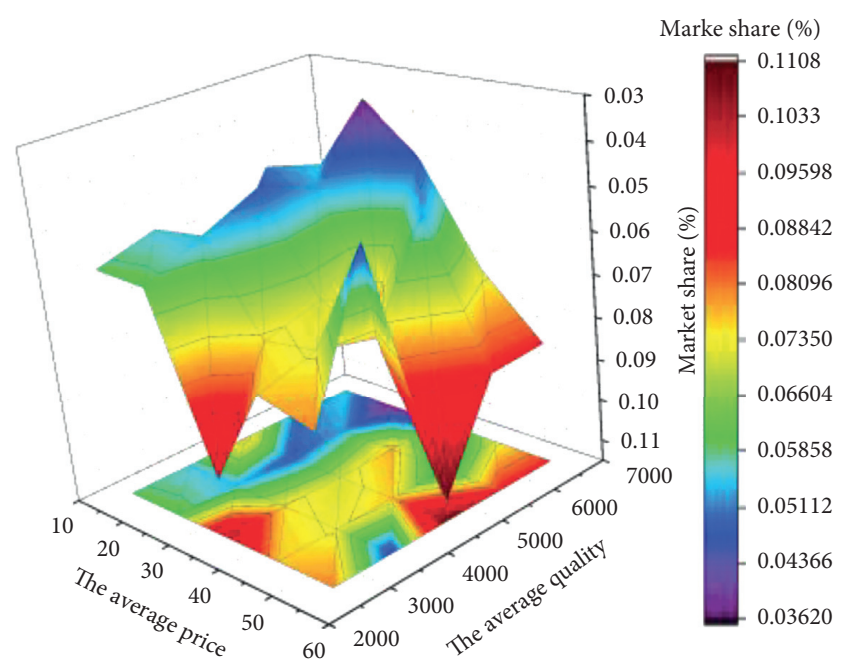

(a)

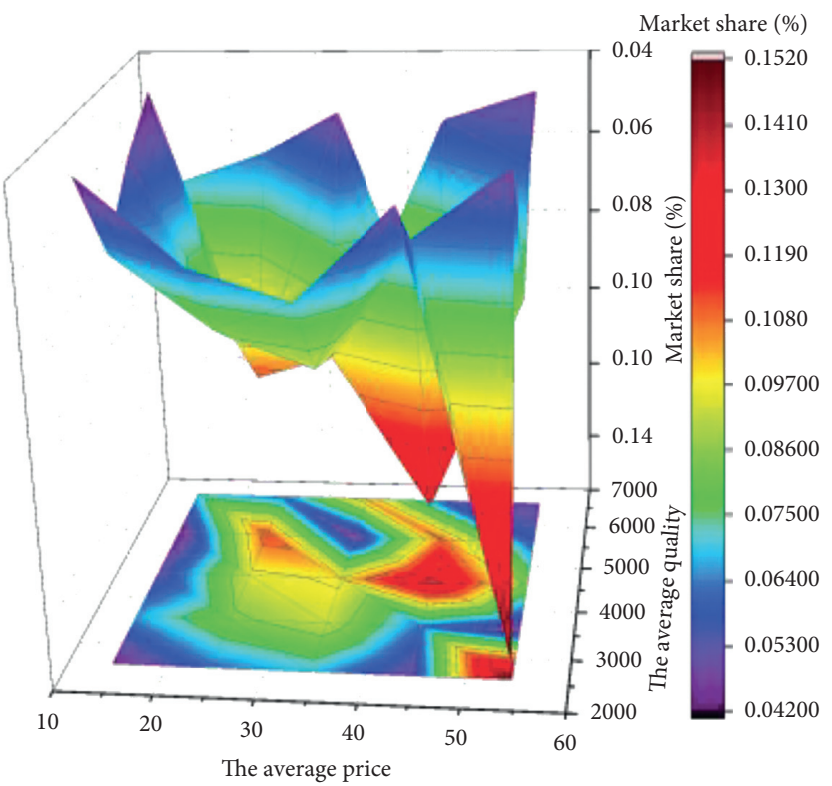

(c)

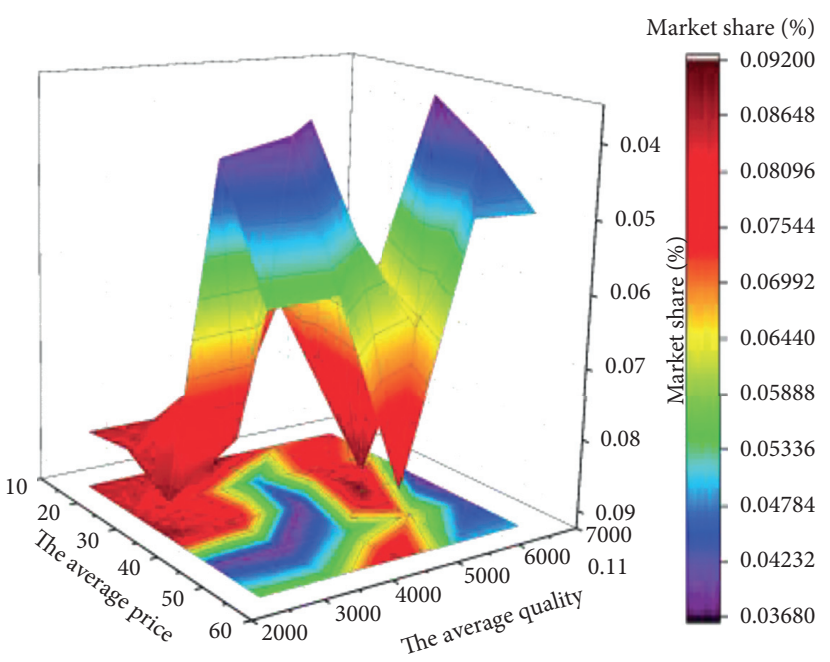

(b)

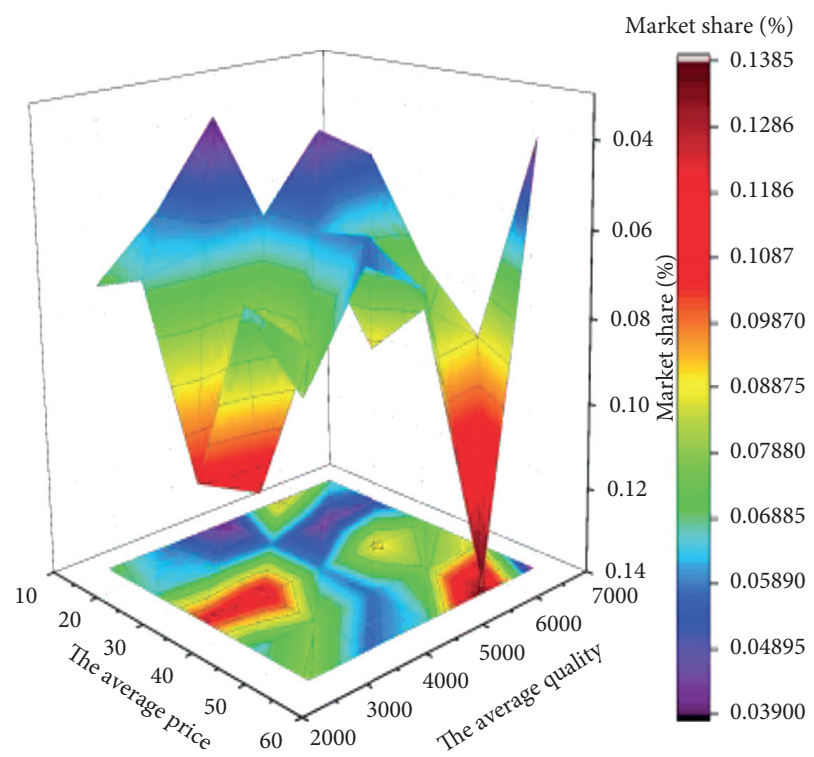

(d)

FIGURE 11: Influence of average quality and price on market share based on different network topologies.

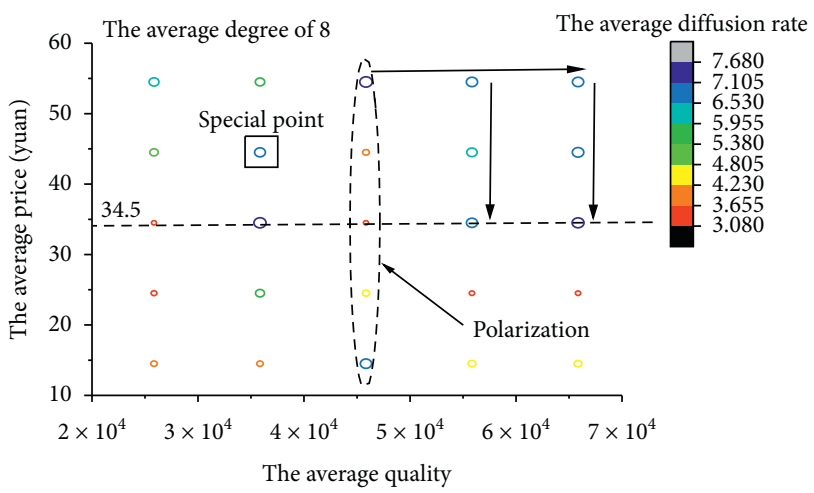

FIGURE 12: Influence of average quality and price on the mean diffusion rate with an average degree of 8 . diffusion theory [49] and the subject-based theoretical criteria [11].

To ensure internal effectiveness, the software was thoroughly tested during implementation to ensure compliance with the specified formal model.

We conducted extensive unit and integration tests and performed simulations of extreme parameter settings to verify whether they produced the expected results (e.g., diffusion failed at very high price levels if the network was too large on average). As a first step toward establishing microvalidity, the survey data used for parameterization were examined carefully. By showing the recorded agent's individual behavior (e.g., communication behavior and purchase decision), the results were found to be consistent with the survey and settings. 


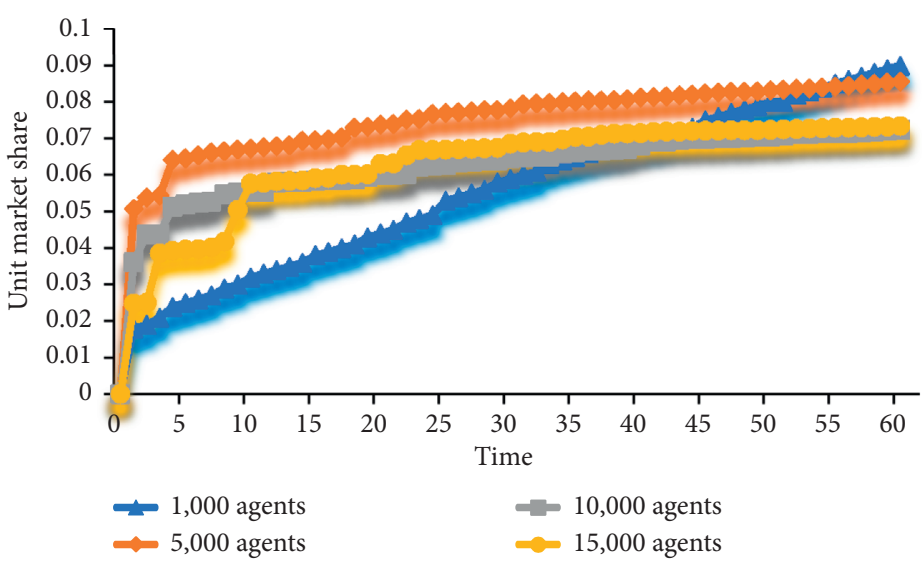

FIGURE 13: Sensitivity analysis for the scale of consumer agents.

TABLE 4: The change of probability distribution threshold.

\begin{tabular}{|c|c|c|c|c|c|}
\hline & $\mathrm{X}_{0}^{*}(i, t)$ & $\mathrm{X}_{1}^{*}(i, t)$ & $\mathrm{X}_{2}^{*}(i, t)$ & $\mathrm{X}_{3}^{*}(j, t)$ & $\mathrm{X}_{4}^{*}(j, t)$ \\
\hline Reference group & Uniform $(0.3,0.8)$ & Uniform $(0.8,1)$ & Uniform $(0,0.3)$ & Uniform $(0.5,1)$ & Uniform $(0,0.5)$ \\
\hline Group 1 & Uniform $(0.3,0.7)$ & Uniform $(0.7,1)$ & Uniform $(0,0.3)$ & Uniform $(0.5,1)$ & Uniform $(0,0.5)$ \\
\hline Group 2 & Uniform $(0.2,0.8)$ & Uniform $(0.8,1)$ & Uniform $(0,0.2)$ & Uniform $(0.5,1)$ & Uniform $(0,0.5)$ \\
\hline Group 3 & Uniform $(0.3,0.9)$ & Uniform $(0.9,1)$ & Uniform $(0,0.3)$ & Uniform $(0.5,1)$ & Uniform $(0,0.5)$ \\
\hline Group 4 & Uniform $(0.1,0.8)$ & Uniform $(0.8,1)$ & Uniform $(0,0.1)$ & Uniform $(0.5,1)$ & Uniform $(0,0.5)$ \\
\hline Group 5 & Uniform $(0.3,0.8)$ & Uniform $(0.8,1)$ & Uniform $(0,0.3)$ & $\operatorname{Uniform}(0.7,1)$ & Uniform $(0,0.7)$ \\
\hline Group 6 & Uniform $(0.3,0.8)$ & Uniform $(0.8,1)$ & Uniform $(0,0.3)$ & Uniform $(0.9,1)$ & Uniform $(0,0.9)$ \\
\hline
\end{tabular}

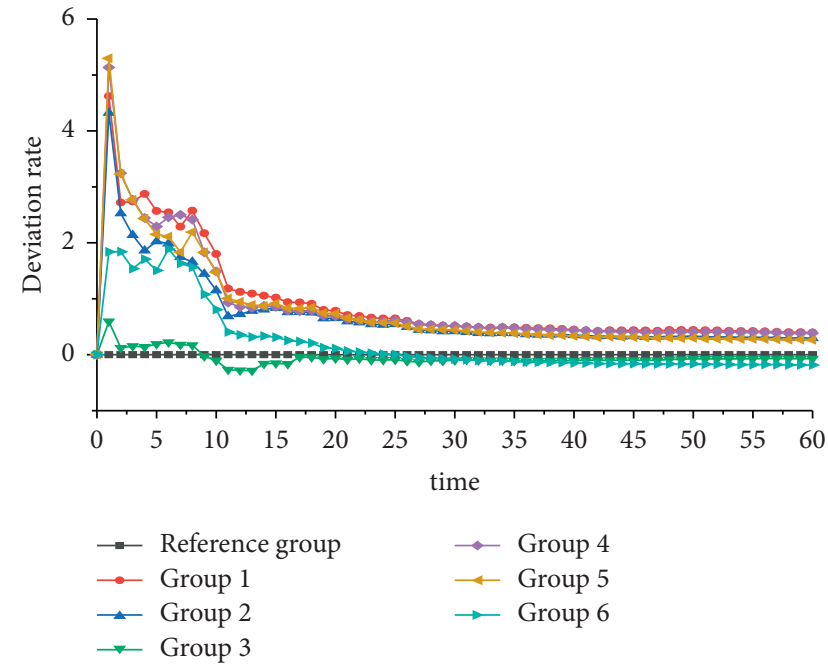

FIGURE 14: Sensitivity analysis of probability distribution threshold.

Macroverification of the new product diffusion model is difficult because real diffusion data can only be obtained after products into the market. For the simulation program in this study, we first considered the macroscopic level of results verification. The simulation results reproduce wellknown procedural facts, such as the typical S-shaped curve. However, as films are short life cycle products, their take-off stage is very short, and so the beginning of the curve and the growth stage are almost connected. In addition, the market share at the steady state is in line with the expectation of the relevant data collected.
By comparing the results of this study with the widely used converged Bass model, cross-model validation was carried out. The Bass model was fitted into the diffusion curves of the three average price levels using the nonlinear least squares method. Overall, we found that the Bass model is quite consistent with the simulation output $\left(R_{\tilde{p}=24.5}^{2}=0.94, R_{\tilde{p}=34.5}^{2}=0.72, R_{\tilde{p}=44.5}^{2}=0.97\right)$, and we concluded that the simulation reproduces the stylized facts embodied in the Bass model.

\section{Conclusion}

In this study, we built a new coupling model based on the combination of the improved SEIR model and HAM. This model can be used to analyze the diffusion dynamic mechanism of new products under consumer heterogeneity, and the parameterization of the model is shown based on experimental data. Our efforts aimed to clearly and strictly consider consumer heterogeneity in all aspects in a simplified consumer purchase decision-making framework that affects consumers' purchase behavior at the individual level. Specifically, in our model, determinants of purchasing probability at the individual level include key determinants of the consumers' preference (e.g., sensitivity to price and quality and the degree of risk aversion), network topology environment, initial perception, and perceived reliability regarding information flow. These determinants can be measured using individual-level statistical data before entering the market with new products, as shown in the pilot study in Sections 4 and 5. 
Further innovations in our model include phased decision-making, building a consumer network approach, considering the combination of consumer behavior with different characteristics, and providing the opportunity to model a variety of marketing mix variables. This is important for online marketers because they may need to use different marketing strategies to effectively promote their products according to the nature of the products and characteristics of consumers.

The influence of consumer heterogeneity on the scale and speed of new product diffusion cannot be fully captured by existing models because it needs to consider the characteristics and behavioral combinations of individual consumers. The behavior combinations of individual consumers in this study are based on the transformation probability of the improved SEIR model. In this model, the transformation probability is determined by three consumer heterogeneity characteristics: consumer sensitivity heterogeneity, consumer heterogeneity caused by network topology, and information flow.

The heterogeneity of consumer sensitivity determines the probability of target consumers buying new products. Consumer heterogeneity caused by network topology determines the probability that potential consumers are connected to purchasing consumers. This probability, combined with the crowd effect and interaction effect, jointly determines the probability that potential consumers will purchase in the network environment. Consumer heterogeneity caused by information flow determines the probability of potential consumers' rational purchases in the context of constantly updated information. Therefore, this study concludes that the probability basis for individual consumers to make purchase decisions is $X_{g}(i, t)+\Theta(t) \xi_{i} \delta_{i}+P(t \mid \Gamma, \Psi)$. The coupling model framework based on the improved SEIR model and HAM is flexible. Due to the conceptualization of the purchase process, it provides a behavioral basis for the interpretation of various diffusion nonlinear dynamics.

We believe that building a new product diffusion dynamics model is not the only contribution of this study. Other main objectives of our research were to demonstrate the likely impact of consumer heterogeneity on the diffusion of new products and to obtain real-world data in sufficient detail to provide an empirical basis for such a detailed and complex model. By using specific instantiated product films, we were able to demonstrate that the need for detailed microdata could indeed be met, and this simulation could actually be based on empirical data. We see this as an important step in moving from a conceptual model to a realworld application.

However, there are still some limitations and challenges. Although we established the model based on perfect theory as much as possible, we can further refine all aspects of the model. The proposed model can be parameterized, extended, and modified in various ways to reflect the difference in individual consumers' decision-making behavior, but this type of extension needs more empirical research and theoretical development. Because of the significant influence of social structure on the diffusion model, future empirical research should solve the difference in connection weight between consumers and the influence of product type on the network structure, and then determine the network model suitable for various possible applications. Finally, our simulation experiment only simulated the results of the given scenarios. Embedding the constructed model into a simulation optimization framework to support decision makers in determining the optimal strategy would be a challenging but worthwhile research work for the future.

\section{Data Availability}

All movie-related data in this study are available from the corresponding author upon request.

\section{Conflicts of Interest}

The authors declare that they have no conflicts of interest.

\section{Acknowledgments}

Financial support from the National Nature Science Foundation of China under Grant no. 71672004 is gratefully acknowledged.

\section{References}

[1] J. Shim and M. Bliemel, "Ignition of new product diffusion in entrepreneurship: an agent-based approach," Entrepreneurship Research Journal, vol. 8, no. 2, pp. 1-18, 2018.

[2] S. Li, Z. Jin, and Z. Jin, "Modeling and analysis of new products diffusion on heterogeneous networks," Journal of Applied Mathematics, vol. 2014, Article ID 940623, 12 pages, 2014.

[3] E. Kiesling, M. Günther, C. Stummer, and L. M. Wakolbinger, "Agent-based simulation of innovation diffusion: a review," Central European Journal of Operations Research, vol. 20, no. 2, pp. 183-230, 2012.

[4] S. Fabian, J. Simon, and B. Thomas, "A review of designing empirically grounded agent-based models of innovation diffusion: development process, conceptual foundation and research agenda," Contributions from the Institute for Infrastructure and Resource Management, vol. 1, pp. 1-22, 2019.

[5] H. Zhang and Y. Vorobeychik, "Empirically grounded agentbased models of innovation diffusion: a critical review," Artificial Intelligence Review, vol. 52, no. 1, pp. 707-741, 2019.

[6] C. Stummer, E. Kiesling, M. Günther, and R. Vetschera, "Innovation diffusion of repeat purchase products in a competitive market: an agent-based simulation approach," European Journal of Operational Research, vol. 245, no. 1, pp. 157-167, 2015.

[7] B. Wu and T. Duan, "Nonlinear dynamics characteristic of risk contagion in financial market based on agent modeling and complex network," Complexity, vol. 2019, Article ID 2946018, 12 pages, 2019.

[8] Y. Gong and F. Li, "A review of new product diffusion models based on individual consumer adoption decisions," Science and Technology Management Research, vol. 40, no. 6, pp. 239-242, 2007. 
[9] S. Fay and J. Xie, "The economics of buyer uncertainty: advance selling vs. Probabilistic selling," Marketing Science, vol. 29, no. 6, pp. 1040-1057, 2010.

[10] X. Chen, X. Han, and H. Ma, "Sales strategy of information goods under consumer heterogeneity influence,"Systems Engineering-Theory \& Practice, vol. 37, no. 8, pp. 2111-2118, 2017.

[11] W. Rand and R. T. Rust, "Agent-based modeling in marketing: guidelines for rigor," International Journal of Research in Marketing, vol. 28, no. 3, pp. 181-193, 2011.

[12] S. Yi and J.-H. Ahn, "Managing initial expectations when word-of-mouth matters," European Journal of Marketing, vol. 51, no. 1, pp. 123-156, 2017.

[13] I. Song and P. K. Chintagunta, "A micromodel of new product adoption with heterogeneous and forward-looking consumers: application to the digital camera category," Quantitative Marketing and Economics, vol. 1, no. 4, pp. 371-407, 2003.

[14] L. Jun, W. Jiepeng, Z. Yongle, and C. Tingqiang, "Scare behavior diffusion model of health food safety based on complex network," Complexity, vol. 2018, Article ID 5902105, 14 pages, 2018.

[15] K. Khashanah and T. Alsulaiman, "Connectivity, information jumps, and market stability: an agent-based approach," Complexity, vol. 2017, no. 8, Article ID 6752086, 16 pages, 2017.

[16] Y. K. Hessary and M. Hadzikadic, "Role of behavioral heterogeneity in aggregate financial market behavior: an agentbased approach," Procedia Computer Science, vol. 108, pp. 978-987, 2017.

[17] K. Khashanah and T. Alsulaiman, "Network theory and behavioral finance in a heterogeneous market environment," Complexity, vol. 21, no. S2, pp. 530-554, 2016.

[18] Q. Meng, P. Shen, and Z. Li, "Diffusion of green products: considering the double effects of "decoy" and "herd," in Proceedings of the 2018 International Conference on Information Management \& Management Science-IMMS '18, Chengdu, China, August 2018.

[19] J. Lin, Y. Li, X. Yue, and G. Nie, "Research on multi-agent simulation on new product diffusion under different network environment," Industrial Technology \& Economy, vol. 37, no. 7, pp. 3-9, 2018.

[20] R. A. Chatterjee and J. Eliashberg, "The innovation diffusion process in a heterogeneous population: a micromodeling approach," Management Science, vol. 36, no. 9, pp. 1057-1079, 1990.

[21] A. Derbali and S. Hallara, "Measuring systemic risk of Greek banks: new approach by using the epidemic model "SEIR"," Cogent Business \& Management, vol. 3, no. 1, Article ID 1153864, 2016.

[22] Y. Zhou, C. Wu, Q. Zhu, Y. Xiang, and S. W. Loke, "Rumor source detection in networks based on the SEIR model," IEEE Access, vol. 7, pp. 45240-45258, 2019.

[23] Q. Zhou, S. Sun, and Q. Liu, "The capital flow of stock market studies based on epidemic model with double delays," Physica A: Statistical Mechanics and Its Applications, vol. 526, Article ID 120733, 2019.

[24] L. Jain, R. Katarya, and S. Sachdeva, "Role of opinion leader for the diffusion of products using epidemic model in online social network," in Proceedings of the 2019 Twelfth International Conference on Contemporary Computing (IC3), pp. 1-6, Noida, India, August 2019.

[25] Q. Huang and Y. Zhang, "Two competitive products diffusion in heterogeneous consumer social networks with repeat purchase," Advances in Difference Equations, vol. 2019, no. 1, p. 309, 2019.
[26] P. Jiang, X. Yan, and L. Wang, "A viral product diffusion model to forecast the market performance of products," Discrete Dynamics in Nature and Society, vol. 2017, no. 2, Article ID 9121032, 10 pages, 2017.

[27] P. Shao and P. Hu, "Product diffusion using advance selling strategies: an online social network perspective," Journal of Artificial Societies and Social Simulation, vol. 20, no. 2, pp. 1-15, 2017.

[28] G. Fibich, "Bass-SIR model for diffusion of new products in social networks," Physical Review E, vol. 94, no. 3, Article ID 032305, 2016.

[29] H. Rahmandad and J. Sterman, "Heterogeneity and network structure in the dynamics of diffusion: comparing agent-based and differential equation models," Management Science, vol. 54, no. 5, pp. 998-1014, 2008.

[30] R. J. Hill, "Belief, attitude, intention and behavior: an introduction to theory and research. By martin fishbein; icek ajzen," Philosophy \& Rhetoric, vol. 41, no. 4, pp. 842-844, 1975.

[31] W. Huiru, S. Jinhui, F. Jianying, F. Huiru, Z. Zhijian, and M. Weisong, "An agent-based modeling and simulation of consumers' purchase behavior for wine consumption," IFACPapersOnLine, vol. 51, no. 17, pp. 843-848, 2018.

[32] D. J. Mcfarland, "Motivationalcontrol systems," in Book Motivational Control Systems, D. J. McFarland, Ed., Academic Press, Cambridge, MA, USA, 1974.

[33] C. Li, Z. Wei, and Y. Quan, "A unified framework for recommending items, groups and friends in social media environment via mutual resource fusion," Expert Systems with Applications, vol. 40, no. 8, pp. 2889-2903, 2013.

[34] H.-H. Ku, "Consumer affects when making undesirable purchases to meet the minimum purchase requirement: decision-related variables as moderators," Journal of Consumer Behaviour, vol. 18, no. 1, pp. 53-62, 2019.

[35] V. Steenburg and Naderi, "Unplanned purchase decision making under simultaneous financial and time pressure," Journal of Marketing Theory and Practice, vol. 28, no. 1, pp. 98-116, 2020.

[36] T. Zhang and D. Zhang, "Agent-based simulation of consumer purchase decision-making and the decoy effect," Journal of Business Research, vol. 60, no. 8, pp. 912-922, 2007.

[37] R. B. B Kim and P. E. Rossi, "Modeling the distribution of price sensitivity and implications for optimal retail pricing," Journal of Business \& Economic Statistics, vol. 13, no. 3, 1995.

[38] J. Kunegis, M. Blattner, and C. Moser, "Preferential attachment in online networks: measurement and explanations," pp. 205-214, 2013.

[39] A.-L. Barabási and R. Albert, "Emergence of scaling in random networks," Science, vol. 286, no. 5439, pp. 509-512, 1999.

[40] B. Hajek and S. Sankagiri, "Community recovery in a preferential attachment graph," IEEE Transactions on Information Theory, vol. 65, no. 11, pp. 6853-6874, 2019.

[41] S. Han, M. Sun, B. C. Ampimah, and D. Han, "Epidemic spread in bipartite network by considering risk awareness," Physica A: Statistical Mechanics and Its Applications, vol. 492, pp. 1909-1916, 2018.

[42] J. H. Roberts and G. L. Urban, "Modeling multiattribute utility, risk, and belief dynamics for new consumer durable brand choice," Management Science, vol. 34, no. 2, pp. 167-185, 1988.

[43] W. L. Hays and R. L. Winkler, "Statistics: probability, inference, and decision," Technometrics, vol. 13, no. 4, p. 920, 1971.

[44] D. R. Cox and H. D. Miller, "The theory of stochastic processes," Applied Statistics, vol. 16, no. 2, p. 177, 1967. 
[45] H. Cui and Y. Cheng, "Combinatorial optimization of free size and advertising intensity of online content in mixed-revenue mode," Journal of Systems \&Management, vol. 28, no. 5, pp. 946-954, 2019.

[46] Y. Tang, J. Yan, and F. Yu, "Multi-product firms, heterogeneous quality and exporting behaviour: product-level evidence from the movie industry," Applied Economics, vol. 50, no. 54, pp. 5920-5934, 2018.

[47] D. A. Reinstein and C. M. Snyder, "The influence of expert reviews on consumer demand for experience goods: a case study of movie critics*," Journal of Industrial Economics, vol. 53, no. 1, pp. 27-51, 2005.

[48] R. Peres, E. Muller, and V. Mahajan, "Innovation diffusion and new product growth models: a critical review and research directions," International Journal of Research in Marketing, vol. 27, no. 2, pp. 91-106, 2010.

[49] Rogers and M. Everett, "Diffusion of innovations," Book Diffusion of Innovations, pp. 97-107, Springer Fachmedien Wiesbaden, Berlin, Germany, 2016. 